\title{
Relativistic quantum chaos-An emergent interdisciplinary field
}

Ying-Cheng Lai, Hong-Ya Xu, Liang Huang, and Celso Grebogi

Citation: Chaos 28, 052101 (2018); doi: 10.1063/1.5026904

View online: https://doi.org/10.1063/1.5026904

View Table of Contents: http://aip.scitation.org/toc/cha/28/5

Published by the American Institute of Physics

\section{Articles you may be interested in}

When chaos meets relativistic quantum mechanics

Scilight 2018, 180006 (2018); 10.1063/1.5037088

Hybrid forecasting of chaotic processes: Using machine learning in conjunction with a knowledge-based model Chaos: An Interdisciplinary Journal of Nonlinear Science 28, 041101 (2018); 10.1063/1.5028373

Accurate detection of hierarchical communities in complex networks based on nonlinear dynamical evolution Chaos: An Interdisciplinary Journal of Nonlinear Science 28, 043119 (2018); 10.1063/1.5025646

Using machine learning to replicate chaotic attractors and calculate Lyapunov exponents from data Chaos: An Interdisciplinary Journal of Nonlinear Science 27, 121102 (2017); 10.1063/1.5010300

Optimal control of networks in the presence of attackers and defenders

Chaos: An Interdisciplinary Journal of Nonlinear Science 28, 051103 (2018); 10.1063/1.5030899

Impacts of opinion leaders on social contagions

Chaos: An Interdisciplinary Journal of Nonlinear Science 28, 053103 (2018); 10.1063/1.5017515

\section{PHYSICS TODAY}

MANAGER'S GUIDE WHITEPAPERS
Accelerate R\&D with Multiphysics Simulation

\section{READ NOW}

PRESENTED BY

マ๐ $\subset$ MSOL 


\title{
Relativistic quantum chaos-An emergent interdisciplinary field
}

\author{
Ying-Cheng Lai, ${ }^{1,2, a)}$ Hong-Ya Xu, ${ }^{1}$ Liang Huang, ${ }^{3}$ and Celso Grebogi ${ }^{4}$ \\ ${ }^{1}$ School of Electrical, Computer and Energy Engineering, Arizona State University, Tempe, Arizona 85287, \\ USA \\ ${ }^{2}$ Department of Physics, Arizona State University, Tempe, Arizona 85287, USA \\ ${ }^{3}$ School of Physical Science and Technology, and Key Laboratory for Magnetism and Magnetic Materials of \\ MOE, Lanzhou University, Lanzhou, Gansu 730000, China \\ ${ }^{4}$ Institute for Complex Systems and Mathematical Biology, King's College, University of Aberdeen, \\ Aberdeen AB24 3UE, United Kingdom
}

(Received 25 February 2018; accepted 4 April 2018; published online 3 May 2018)

\begin{abstract}
Quantum chaos is referred to as the study of quantum manifestations or fingerprints of classical chaos. A vast majority of the studies were for nonrelativistic quantum systems described by the Schrödinger equation. Recent years have witnessed a rapid development of Dirac materials such as graphene and topological insulators, which are described by the Dirac equation in relativistic quantum mechanics. A new field has thus emerged: relativistic quantum chaos. This Tutorial aims to introduce this field to the scientific community. Topics covered include scarring, chaotic scattering and transport, chaos regularized resonant tunneling, superpersistent currents, and energy level statistics - all in the relativistic quantum regime. As Dirac materials have the potential to revolutionize solid-state electronic and spintronic devices, a good understanding of the interplay between chaos and relativistic quantum mechanics may lead to novel design principles and methodologies to enhance device performance. Published by AIP Publishing.

https://doi.org/10.1063/1.5026904
\end{abstract}

The characteristic difference between nonrelativistic and relativistic quantum systems lies in the energy-momentum relation, which is parabolic for the former and linear (hyperbolic) for the latter, as described by the Schrödinger and the massless (massive) Dirac equations, respectively. The traditional field of quantum chaos deals with the quantum manifestations or fingerprints of classical chaos, but previous efforts were mostly confined to nonrelativistic quantum systems. To study the unique physics of classical chaos in relativistic quantum systems is fundamental with potentially significant applications. This is so because of a frontier area of tremendous current interest: Dirac materials, examples of which include graphene, topological insulators, molybdenum disulfide, and topological Dirac semimetals. A common feature of these materials is that the low energy excitations obey relativistic quantum mechanics, leading to physical properties that are not usually seen in conventional semiconductor materials. For example, graphene, a single, one-atom-thick sheet of carbon atoms arranged in a honeycomb lattice, was experimentally realized in 2004. Due to its peculiar hexagonal lattice structure, the band structure exhibits a linear dependence of the energy on the momentum about a Dirac points, signifying relativistic motion. Devices made of graphene are potentially capable of operating at much higher speed than those based on conventional semiconductor materials. The purpose of this Tutorial is to introduce the emergent field of relativistic quantum chaos to the scientific community, covering a number of topics including scarring, transport, scattering, resonant tunneling, persistent currents, and energy level spacing statistics. An emphasis will be on the

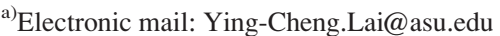

behaviors that are uniquely relativistic quantum, such as chiral scars and phenomena due to Klein tunneling and peculiar boundary modes, i.e., those that do not occur in the counterpart, nonrelativistic quantum systems.

\section{INTRODUCTION}

The development of chaos theory has fundamentally changed our understanding of a large variety of nonlinear dynamical phenomena. The question of what chaos can do to a quantum system led to the field of quantum chaos. Quantum systems are fundamentally linear and obey the superposition principle, so, in general, chaos, or sensitive dependence on initial conditions, cannot be expected. The field of quantum chaos thus aims to uncover the quantum manifestations or fingerprints of classical chaotic behaviors in the semiclassical limit. ${ }^{1,2}$ (It should be noted that the classical phase space distributions obey the superposition principle, because the Liouville equation is linear. The important difference is that, in quantum mechanics, the "notion of a phase space trajectory loses its meaning."2) In the field of quantum chaos, depending on whether the system under study is closed or open, different issues have been pursued. For example, in closed chaotic Hamiltonian systems, the basic phenomena that have been and continue to be studied include energy level-spacing statistics ${ }^{3-19}$ and quantum scarring. ${ }^{20-52}$ In open Hamiltonian systems, quantum chaotic scattering has been investigated extensively. ${ }^{53-68}$ In addition, the phenomena of quantum diffusion and wavefunction localization in classically driven chaotic systems have been studied. ${ }^{69-84}$ 
Pioneers in the traditional field of quantum chaos include Chirikov, Casati, Shepelyansky, Guarneri, Izrailev, Bohigas, Kaufman, Berry, Smilansky, Prange, Fishman, Heller, among others. Especially, Chirikov conceived and studied the chaotic properties of the standard map, ${ }^{77,85}$ which has been a paradigm in the study of Hamiltonian systems and quantum chaos with ubiquitous applications to a large variety of physical systems. Bohigas and collaborators uncovered the phenomenon of level repulsion and attributed its origin to classical chaos. ${ }^{3}$ Casati, Chirikov, Guarneri, and Shepelyansky made significant contributions to our understanding of energy level statistics ${ }^{6,9}$ and quantum behaviors of classically driven chaotic systems, a representative one being the ionization of excited hydrogen atoms by microwave fields. ${ }^{70-76,78-82,86,87}$ Kaufman and Heller discovered quantum scarring. ${ }^{20,21,23,47,88}$ Prange and Fishman uncovered the remarkable phenomenon of quantum localization in kicked quantum chaotic systems. ${ }^{69,89,90}$ Smilansky pioneered the area of quantum chaotic scattering. ${ }^{11,17,53,54,60,61,66,91} \mathrm{Sir}$ Michael Berry made many seminal contributions to the fundamentals of quantum chaos. ${ }^{4,7,26,92}$

Vast majority of the studies on quantum chaos in the past were for nonrelativistic quantum systems described by the Schrödinger equation, which possesses a parabolic relation between the energy and momentum. However, there were early studies on the dynamics of relativistic kicked rotors (the so-called solvable Maryland model). ${ }^{93-95}$ Berry and Mondragon were the first to study (about thirty years ago) the energy level spacing statistics of a classically chaotic system in the relativistic quantum regime-the neutrino billiard system governed by the massless Dirac equation ${ }^{96}$ For such a system, the energy is linearly proportional to the momentum or the wave vector. In a subsequent work by Antoine et al. ${ }^{97}$ a 2D fermion billiard in a curved space coupled with a magnetic field was studied. Results on energy levels were obtained under a more generalized set of boundary conditions than those treated by Berry and Mondragon. ${ }^{96}$ The authors verified ${ }^{97}$ that, under the same boundary conditions, both their work and that of Berry and Mondragon gave the same results. In Ref. 98, Tomaschitz investigated chaos in open Robertson-Walker cosmologies and found localized wave fields, which are solutions of the Klein-Gordon equation quantized on the bounded trajectories associated with the classical geodesic motion. Based on the minisuperspace model described by the Wheeler-DeWitt equation, there were studies on chaos in quantum cosmology with an emphasis on the breakdown of the WKB approximation and relativistic wave packet dynamics. ${ }^{99-101}$ In addition, there were general discussions about the spectral statistics of the Dirac operator on lattices or graphs in terms of the random matrix theory. ${ }^{102-104}$ In spite of these studies, ${ }^{97,98,105}$ not much effort was devoted to the study of the interplay between chaos and relativistic quantum mechanics for a long period of time after the pioneering work of Berry and Mondragon. ${ }^{96}$

Recent years have witnessed a rapid growth of interest in Dirac materials ${ }^{106,107}$ such as graphene,${ }^{108-114}$ topological insulators, ${ }^{115}$ molybdenum disulfide $\left(\mathrm{MoS}_{2}\right){ }^{116,117}$ HITP $\left[\mathrm{Ni}_{3}(\mathrm{HITP})_{2}\right]$ (a combination of nickel and an organic compound called HITP), ${ }^{118}$ and topological Dirac semimetals. ${ }^{19,120}$ A common feature of these materials is that their energy bands contain a Dirac cone structure, so they are described by the Dirac equation, generating phenomena that are not usually seen in conventional metallic or semiconductor materials. For example, from the standpoint of quantum transport, the Dirac cone structure and the resulting pseudospin characteristics of the underlying quasiparticles can lead to unconventional physical properties/ phenomena such as high carrier mobility, anti-localization, chiral tunneling, and negative refractive index. This has important consequences. At the level of basic science, a new field has emerged: Relativistic Quantum Chaos (RQC), which aims to uncover, understand, and exploit relativistic quantum manifestations of classical nonlinear dynamical behaviors including chaos. ${ }^{121-153}$ Topics studied so far include relativistic quantum scarring, ${ }^{121,134,136,153}$ energy level spacing statistics in graphene systems, ${ }^{123,125-127,130}$ relativistic quantum chaotic scattering and transport, ${ }^{128,131,139,142,145,149,150,154}$ relativistic quantum tunneling, ${ }^{133,141,143}$ effects of chaos on persistent currents in Dirac fermion systems, ${ }^{146,148}$ the role of classical dynamics in confinement of massless Dirac fermions, ${ }^{122,129,135,144}$ the interplay between chaos and spin transport in graphene quantum dot systems, ${ }^{151}$ and the role of many body interactions in chaotic graphene quantum billiards. ${ }^{143,152}$ From an applied point of view, due to the underlying physics being effectively governed by the Dirac equation, purely relativistic quantum phenomena such as Klein tunneling, Zitterbewegung, and pair creations can potentially occur in solid state devices and be exploited for significantly improving or even revolutionizing conventional electronics and spintronics. Investigating the manifestations of classical chaos in relativistic quantum systems is thus important with both fundamental and applied values.

The purpose of this Tutorial is to provide a succinct and elementary review of the emergent field of RQC, introducing it to the scientific community. We focus our discussions on two types of systems: massless Dirac fermion systems and graphene. While the main task is to explain the relativistic quantum manifestations of chaos, in some cases, classically integrable dynamics will also be discussed as a contrast. The specific topics to be covered include scarring, chaotic scattering and transport, resonant tunneling, persistent currents, and energy level spacing statistics-all in the relativistic quantum realm. It should be noted that the topics covered here for the 2D massless (pseudo-) Dirac fermion systems comprise only a small part of the field of relativistic quantum chaos, as new phenomena are continue to be uncovered.

To give an example of the phenomena to be discussed in this Tutorial, we briefly describe quantum scarring, a remarkable phenomenon in quantum systems whose dynamics in the classical limit are chaotic. In general, in the semiclassical regime, a wavefunction can be regarded locally as a superposition of many plane waves. Due to classical chaos, the directions of these plane waves are random. Intuitively, one may anticipate the wave functions to have a uniform concentration in the position space. However, signatures of highly nonuniform distribution of the wavefunction were discovered in 1979 by McDonald and Kaufman, ${ }^{20,23}$ and Heller 
pointed out in 1984 that this is a "scarring" phenomenon ${ }^{21}$ in which the wavefunctions tend to concentrate on paths corresponding to unstable periodic orbits in the classical limit and the eigenfunctions associated with different eigenvalues can focus on different periodic orbits. In solid state electronic transport devices, the quantum scarring states are known as quantum "pointer states," which can have a significant impact on the transport properties such as conductance. Relativistic quantum scars in graphene were uncovered ${ }^{121}$ in 2009 , and a class of uniquely relativistic quantum scars, the so-called chiral scars, ${ }^{136}$ were identified in 2013.

While there were previous studies on chaos in classical relativistic systems, ${ }^{155-159}$ the emergent field of relativistic quantum chaos reviewed in this Tutorial is about the relativistic quantum manifestations of classical chaos. In particular, the study is to uncover how classical chaos manifests itself in relativistic quantum systems as described or approximately described by the Dirac equation. This is parallel to the traditional field of quantum chaos, which is defined as the study of the (nonrelativistic) quantum manifestations of classical chaos.

This Tutorial is outlined as follows. For the phenomenon of scarring, besides demonstrating its occurrence in a closed chaotic graphene billiard, we place emphasis on a class of scars that have no counterparts in nonrelativistic quantum systems - chiral scars, the discovery of which was facilitated by the development of a conformal mapping method to accurately determine a large number of eigenstates for a class of chaotic Dirac fermion billiard systems. We then turn to quantum transport and conductance fluctuations in graphene systems, pointing out that classical chaos can play a similar role to that in nonrelativistic quantum systems: suppressing Fano resonances and smoothing out the fluctuations with energy. The next topic of discussion is regularization of resonant tunneling by chaos. We present evidence that, as in nonrelativistic quantum systems, chaos can greatly suppress the spread in the resonant tunneling rate for any small energy interval in both Dirac fermion and graphene systems. Specific challenges with calculating the resonant tunneling rate in such systems and solutions are pointed out and the effect of a uniquely relativistic quantum phenomenonKlein tunneling, on the behavior of the tunneling rate in the small energy regime is demonstrated. The discussion is then extended briefly to a graphene point contact system in which Klein tunneling can lead to an extreme type of conductance fluctuations. Another topic of discussion is persistent currents (permanent currents without requiring any external voltage source) - a quantum phenomenon caused by the breaking of the time reversal symmetry due to a magnetic flux through a ring domain. With explicit numerical evidence and analytic insights, we demonstrate that, for a nonrelativistic quantum ring domain, boundary deformation leading to classical chaos can diminish the currents but they can sustain in a Dirac ring domain. In fact, due to the unconventional boundary conditions in the Dirac system, a distinct set of whispering gallery modes (WGMs) circulating along the outer or the inner boundary can arise regardless of the boundary deformation, which leads to "superpersistent" currents in chaotic Dirac fermion systems. Potential applications of this phenomenon are pointed out. Finally, we describe the energy level spacing statistics in Dirac fermion and graphene systems in terms of the similarities to and differences from the nonrelativistic quantum counterparts. We conclude this Tutorial by offering a general discussion with an emphasis on the unique interplay between classical chaos and relativistic quantum mechanics and speculating on a number of open problems.

It is worth pointing out an important difference between a Dirac fermion system and a graphene system. The energy band structure of graphene is such that there are two nonequivalent Dirac points in the first Brillouin zone. While a quasiparticle in the vicinity of a Dirac point obeys the same (Dirac) equation as that for a Dirac fermion, in a closed domain, the abrupt edge termination defining the confinement couples the spinor wavefunctions from the two valleys in the momentum space, making them inseparable. As a result, the two-component spinor Dirac equation can describe graphene but only in an approximate sense.

\section{RELATIVISTIC QUANTUM SCARS}

Quantum scarring, in which the wavefunctions tend to concentrate on unstable periodic orbits of a classically chaotic Hamiltonian system, is a fundamental phenomenon in quantum chaos..$^{20,21,23-34,38-43,45-49,51,52,88}$ This phenomenon is surprising because, for a closed Hamiltonian system that exhibits fully developed chaos, typical classical trajectories are ergodic, generating a uniform distribution in both phase space and physical space. Naively, one might think that the corresponding quantum wavefunction distribution should be uniform as well, but detailed computations by McDonald and Kaufman ${ }^{20,23}$ indicated that this is not so. What they found was that the eigenstates can be highly nonuniform with a significant density localized in the vicinity of classical unstable periodic orbits-henceforth the first discovery of this remarkable phenomenon. Later, it was coined the term "scarring" by Heller ${ }^{21}$ with physical insights from a random wave model. A semiclassical theory was subsequently developed by Bogomolny ${ }^{24}$ and Berry, ${ }^{26}$ providing a theoretical understanding of the phenomenon.

The first indication that scarring can occur in relativistic quantum systems was obtained through the study of a chaotic graphene billiard, ${ }^{121}$ where it was discovered that the eigenstates tend to concentrate heavily on classical unstable periodic orbits, as illustrated in Fig. 1. The scars are recurrent when the energy varies such that the phase accumulation along the closed orbit gains a change that is the integer multiple of $2 \pi: \delta k \cdot L=2 \pi l$, where $L$ is the length of the orbit, $\delta k$ is the wavenumber increment, and $l$ is an integer. For a massless relativistic particle, because of the linear dependence of its energy on the wave vector, the recurring scarring states occur at equally spaced values of $E$, which has recently been observed experimentally in a mesoscopic graphene ring system. ${ }^{160}$ This should be contrasted to the case of a nonrelativistic quantum particle, where the energy depends on the wave vector parabolically so that recurrent scars occur at equally spaced values of $\sqrt{E}$. Graphene is, however, fundamentally a lattice system for which a relativistic quantum 


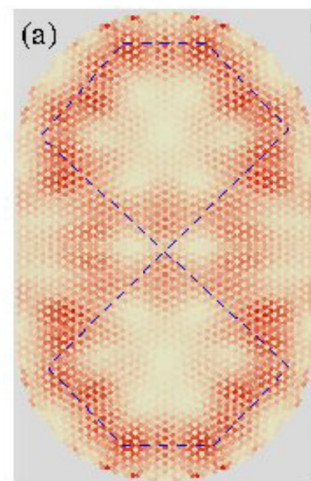

(e)



(b)

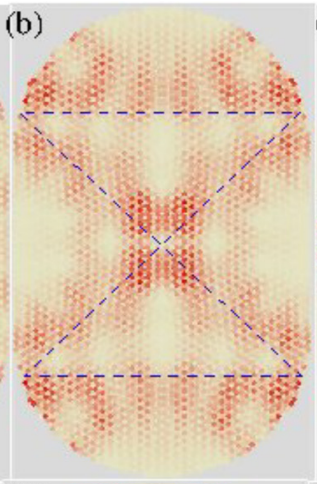

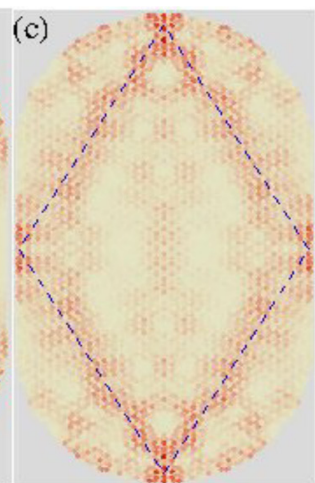

(f)

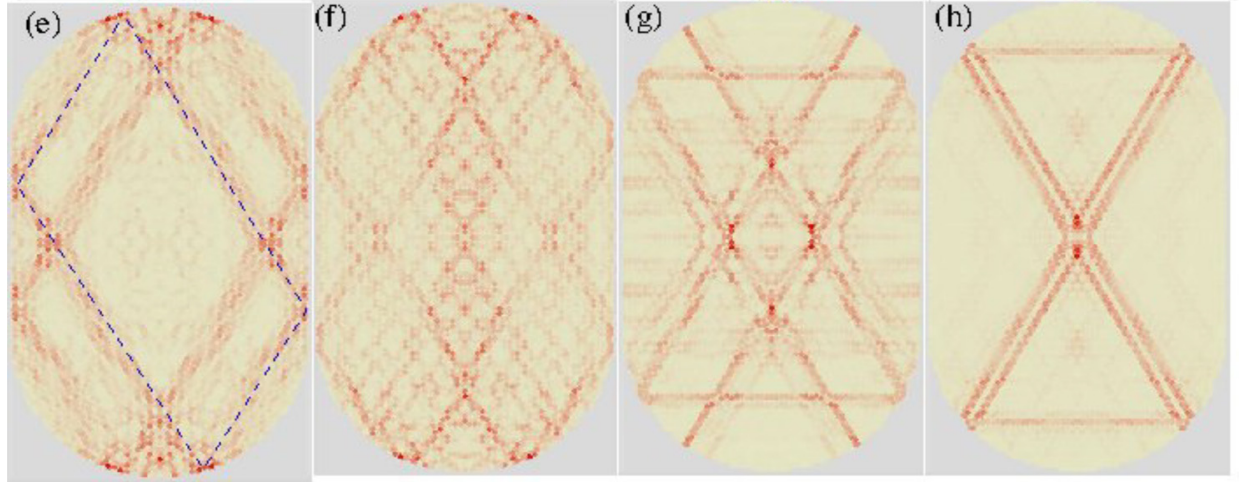

(g)

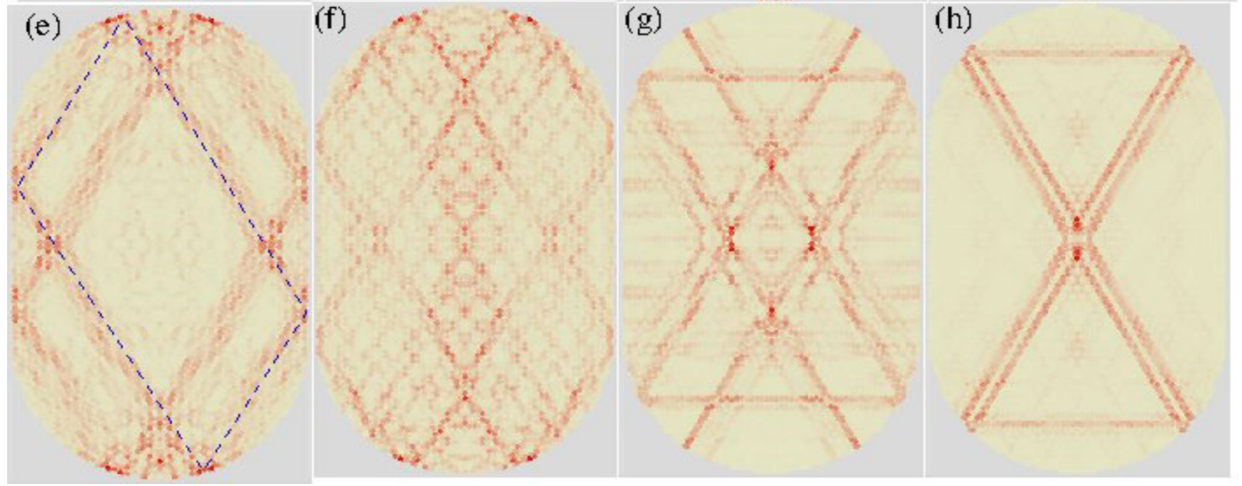

(d)

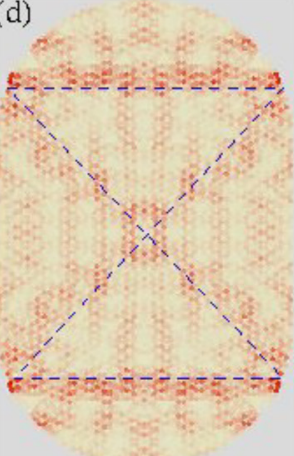

(h)
FIG. 1. Quantum scars in a stadiumshaped chaotic graphene billiard. The billiard domain contains 11814 carbon atoms and has zigzag boundaries along the two straight segments. The energy values for panels (a)-(h) are $E /$ $t=0.25347, \quad 0.36358, \quad 0.57665$, $0.60699,0.81956,0.91061,0.97722$, and 0.99198 , respectively. The dashed lines represent classical periodic orbits. The nearest neighbor hopping energy of graphene is $t \approx 2.8 \mathrm{eV}$ and the lattice constant is $a=2.46 \AA$. The eigenenergies and the eigenstates were calculated using the standard tight-binding Hamiltonian. ${ }^{121}$ description based on the Dirac equation is only approximate. A more serious issue is the existence of two nonequivalent Dirac points ( $\mathbf{K}$ and $\mathbf{K}^{\prime}$ ) in the first Brillouin zone, where the wavefunctions originated from them can couple due to processes such as reflection from the boundary and scattering from impurities. For these reasons, scars in the graphene system are not truly relativistic quantum. Note that, in general, while some eigenstates show scars, the average over an energy interval gives a constant density. That is, the nonscarred states have a somewhat reduced density close to the periodic orbit. As a result, not most eigenstates are scarredonly a subset of them are.

To search for "true" relativistic quantum scars, it is necessary to solve the Dirac equation in two dimensions, demanding accurate solutions in arbitrary confinements that exhibit chaos in the classical limit. (A brief introduction to the Dirac equation in two dimensions together with the boundary conditions is given in the Appendix.) It is also necessary to calculate high energy levels to resolve the fine structures in the spinor wavefunctions so that quantum scars can be accurately identified. Further, a large number of scars are needed so that their statistical properties can be characterized. A finite element method was developed to calculate the eigenstates of Dirac fermions under arbitrary electrical potential profiles, ${ }^{134}$ leading to the discovery that relativistic, spinor type of wavefunctions associated with Dirac fermions can be highly nonuniform in chaotic billiards, and truly relativistic quantum scars indeed exist. However, due to the computational limitation associated with the finite element method, only a handful of the eigenstates can be obtained with high accuracy.

A breakthrough in this area occurred with the development of a conformal mapping method ${ }^{136}$ that enables a very large number (on the order of $10^{4}$ ) of eigenstates of the Dirac equation in a chaotic domain to be calculated with unprecedentedly high accuracy. Examination of the eigenstates revealed a new class of relativistic quantum scars that are characteristically different from those in nonrelativistic quantum systems: chiral scars. ${ }^{136}$ To describe a chiral scar in an intuitive way, we note that, for a conventional quantum scar to reoccur, when a particle traverses one cycle along a scarred orbit that corresponds to a classical unstable periodic orbit, the associated quantum phase change is $2 \pi$. However, for a chiral scar, phenomenologically, it takes two cycles for the phase accumulation of the spinor to be $2 \pi$ and for the scarring state to reappear. This relativistic quantum phenomenon has its origin in the chiral nature of the massless Dirac fermions subject to a massive confinement. More generally, in a chaotic billiard, for quantum scars with an even number of bounces off the billiard wall along a closed orbit, the difference between the total phase accumulation of the wavefunction after one cycle and that of its time-reversed counterpart is an integer multiple of $2 \pi$. However, for relativistic quantum scars with an odd number of bounces, besides the integer multiple of $2 \pi$ phase difference, there will be an extra $\pi$ difference in the total phase accumulation. ${ }^{153}$ As a result, in the semiclassical regime, these orbits cancel each other and disappear in the length spectra.

The success in uncovering chiral scars in relativistic quantum systems can be attributed to the "quasi-analytic" nature of the conformal mapping approach to calculating the eigenstates. In particular, for a broad class of chaotic domains, a proper conformal mapping can transform any such domain to the circular domain for which the solutions of the Dirac equation can be written down analytically. An inverse transform of the solutions thus leads to eigenstates in the original billiard. While a semiclassical understanding of the chiral scar was available, ${ }^{136}$ a complete understanding of this phenomenon was obtained only recently ${ }^{153}$ through a systematic study 
of the effects of an Aharonov-Bohm (AB) flux on the eigenstates of the class of massless Dirac billiards to which the conformal-mapping method is applicable.

We present an intuitive reasoning that leads to the discovery of chiral scars. An examination of a large number of relativistic quantum scars for massless Dirac fermion in a typical chaotic billiard revealed ${ }^{136}$ that, a certain scarring pattern, once having appeared, tends to reappear at a different energy value. This can be understood by resorting to the semiclassical theory, ${ }^{161}$ which states that two repetitive scars associated with the same classical periodic orbit can occur when the action difference satisfies

$$
|\Delta S|=2 \pi n \hbar(n=1,2, \ldots),
$$

where $S=\oint \boldsymbol{p} \mathrm{d} \boldsymbol{q}=\hbar k L$ and $L$ is the length of a given periodic orbit. If one scar has already appeared, say, at $k_{0}$, the eigenfunctions at the wave number $k_{n}=k_{0} \pm n \delta k$ will most likely scar, where $\delta k=2 \pi / L$. It is thus useful to define

$$
\eta(n)=\frac{\left|k_{n}-k_{0}\right|}{\delta k}-\left[\frac{\left|k_{n}-k_{0}\right|}{\delta k}\right],
$$

where $[x]$ denotes the largest integer less than $x$. According to the semiclassical theory for nonrelativistic quantum systems, the quantity $\eta$, by its definition, should exhibit only two distinct values: either close to 0 or to 1 . To calculate the value of $\eta$, some key characteristics of the corresponding scars are needed. In Ref. 136, some of the key features of the calculated scars are listed. Using the data of the most typical types of scars, one can then calculate their values of $\eta(n)$ from Eq. (1). Representative chiral scars are shown in Fig. 2, for which $\eta$ can take on values about $1 / 2$. From the relation $\delta k=2 \pi / L$, one sees that the orbital length becomes $2 L$.

A recent work ${ }^{153}$ has elucidated the physical origin of chiral scars based on considerations of the time reversal symmetry. In particular, in Berry's seminal paper, ${ }^{96}$ timereversal symmetry breaking was interpreted semiclassically as a $\pi$ phase difference between the waves traveling in opposite directions over an orbit with an odd number of reflections off the domain boundary. The chiral scars are those with an odd number of reflections, which differ characteristically from scars with an even number of reflections, as the former have an effective "doubled" orbital length. The physical insight is that, for scars of the Dirac billiard, each bounce at the boundary breaks the time-reversal symmetry, but for orbits of even number of bounces, after circling the orbit, the effects of the bounces at the boundary cancel each other ("even" scars). As noted by Berry and Mondragon, 96 for these orbits, the phase difference of a clockwise orbit and its time-reversal counterpart is an integer multiple of $2 \pi$, thereby preserving the time-reversal symmetry. Only the orbits with an odd number of bounces have the net effect that the phase has a $\pi$ difference in addition to an integer multiple of $2 \pi$, thus breaking the time-reversal symmetry ("odd" scars). A natural question is whether this additional $\pi$ phase difference can be compensated by a magnetic flux. To address this question, it is necessary to solve the Dirac equation for the Aharonov-Bohm (AB) chaotic billiard. The conformal mapping method can again be employed. ${ }^{146}$ Let $S_{+}$ be the classical action associated with a counter-clockwise (a)

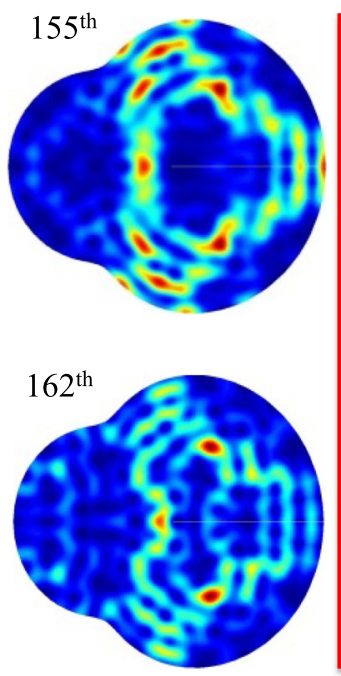

$$
\begin{gathered}
\mathrm{k}_{1}=24.83 \\
\mathrm{k}_{2}=25.43 \\
\left(\mathrm{k}_{2}-\mathrm{k}_{1}\right) / \delta \mathrm{k}=0.46
\end{gathered}
$$

(b)

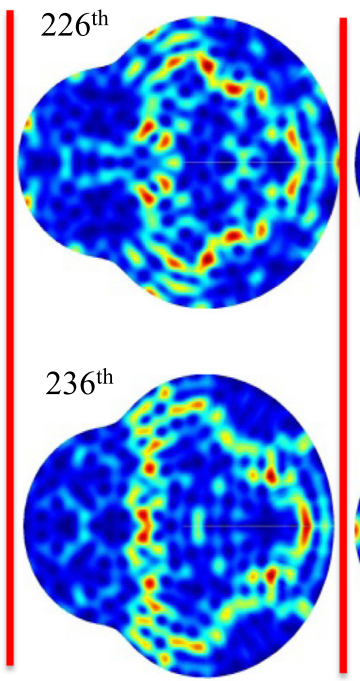

$$
\begin{aligned}
& \mathrm{k}_{1}=30.04 \\
& \mathrm{k}_{2}=30.70
\end{aligned}
$$$$
\left(\mathrm{k}_{2}-\mathrm{k}_{1}\right) / \delta \mathrm{k}=0.50
$$

(c)

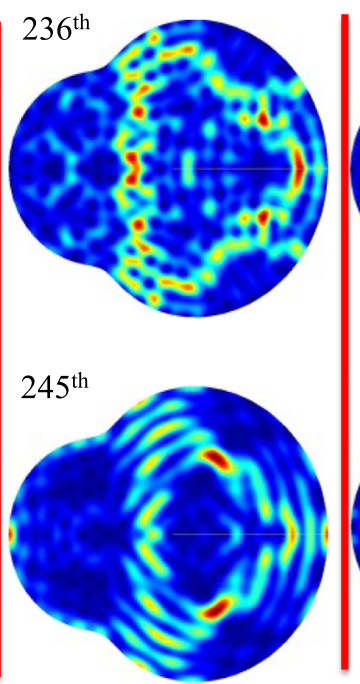

$$
\begin{aligned}
& \mathrm{k}_{1}=30.70 \\
& \mathrm{k}_{2}=31.32
\end{aligned}
$$$$
\left(\mathrm{k}_{2}-\mathrm{k}_{1}\right) / \delta \mathrm{k}=0.48
$$

(d)

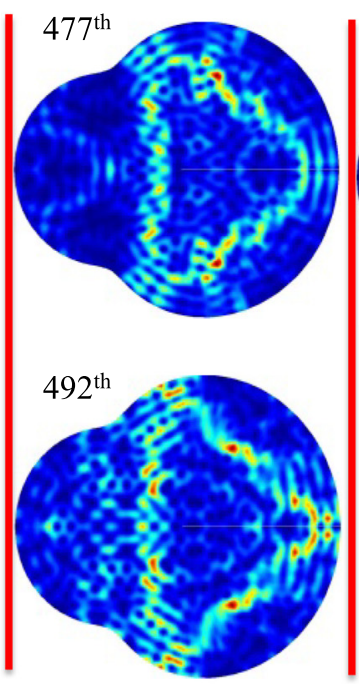

$$
\begin{gathered}
\mathrm{k}_{1}=43.67 \\
\mathrm{k}_{2}=44.31 \\
\left(\mathrm{k}_{2}-\mathrm{k}_{1}\right) / \delta \mathrm{k}=0.50
\end{gathered}
$$

(e)

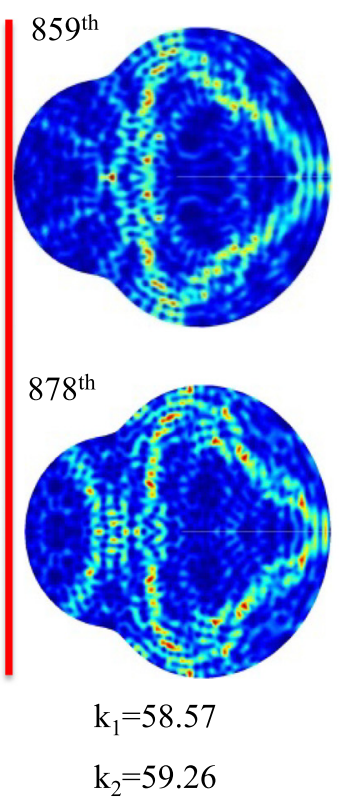

$\left(\mathrm{k}_{2}-\mathrm{k}_{1}\right) / \delta \mathrm{k}=0.53$

FIG. 2. Examples of relativistic quantum chiral scars in a chaotic Dirac billiard system. Ten representative scarring patterns are shown from a pear-shaped Dirac billiard system that is fully chaotic in the classical limit. (a)-(e) The patterns are organized into five pairs in terms of the nearest recurrence, with the corresponding wave vectors listed. For example, in (a), the two eigenstates are the 155th and 162th. For this particular chaotic billiard system, we have $\delta k \approx 1.2959$. The values of the semiclassical indicator $\eta$ as defined in Eq. (1) for all the pairs are also listed, which are about 0.5 , providing evidence for chiral scars that are not predicted by the conventional semiclassical theory. Chiral scars are thus uniquely a relativistic quantum phenomenon. 
orbit and $S_{-}$be its time-reversed counterpart. For odd and even scars, one has $\Delta S=2 \pi(n+1 / 2) \hbar$ and $\Delta S=2 \pi n \hbar$, respectively, where $n$ is an integer. When a magnetic flux $\alpha$ (in units of the magnetic flux quantum $\Phi_{0} \equiv h / e$ ) is present, the action is given by $S=\oint \boldsymbol{p} d \boldsymbol{q}=\hbar(k \cdot L+2 \pi W \alpha)$, where $W$ is the winding number enclosing the flux $(W>0$ for counter-clockwise and $W<0$ for clockwise motions). The difference in action then becomes $\Delta S=2 \pi(n+1 / 2$ $+2 W \alpha) \hbar$ for odd scars and $\Delta S=2 \pi(n+2 W \alpha) \hbar$ for even scars. The magnetic flux can then be used to compensate the $\pi$ phase difference for the odd orbits, which has been verified numerically and analytically. ${ }^{153}$

While relativistic quantum scars in graphene systems have recently been observed experimentally, ${ }^{160}$ at the present, there is no experimental evidence for chiral scars in massless Dirac fermion systems, which requires the setup of a material system with a single Dirac cone and a hard wall confinement of the massive type. The surface states of a 3D topological insulator can be exploited to fulfill such a requirement, where the single Dirac cone structure is realizable $^{162}$ and the massive confinement can be implemented through spatially dependent magnetic doping ${ }^{163}$ or a local exchange coupling with a ferromagnetic insulator cap layer. ${ }^{164,165}$ More specifically, for a 3D topological insulator such as $\mathrm{Bi}_{2} \mathrm{Se}_{3}$, the surface states have a topological origin with a perfect spin-momentum locking, effectively eliminating backscattering from nonmagnetic impurities and generating electronic "highways" with highly efficient transport. The surface states can generally be described by a twodimensional Dirac Hamiltonian. A likely candidate for experimental study of chiral scars is thus 3D topological insulators.

\section{ROLE OF CHAOS IN QUANTUM TRANSPORT}

In a typical scenario of electronic transport, one applies a voltage to a device to drive electrons from one end to another. The device can be a conductor, a semiconductor junction, or a quantum dot, or some others. Consider the simple case of a two-dimensional conductor of length $L$ and width $W$. Classically, the transport process is described by the Ohm's law, which relates the conductance $G$ (or the resistance) to the conductivity $\sigma$ and the geometrical parameters of the conductor as $G=\sigma W / L$. In the classical picture, the movement of an electron can be regarded as that of a biased random walk, where it collides with various lattice atomic sites and random impurities but with a drift towards the higher voltage end. The collisions, which change the momentum of the electron, are effectively scattering events. The average distance between two adjacent scattering events during which the electron momentum is preserved is the mean free path $L_{m}$. (For example, at room temperature, the values of $L_{m}$ for copper and silver are $39.9 \mathrm{~nm}$ and $53.5 \mathrm{~nm}$, respectively. ${ }^{166}$ )

The Ohm's law is valid only if the mean free path is much shorter than the characteristic length scale of the conductor: $L_{m} \ll L$ or $W$. For a device whose size is on the same order of magnitude as or smaller than the mean free path, a quantum mechanical description becomes necessary, where the conductor can effectively be regarded as a waveguide with a number of transverse modes. In this case, one enters the regime of quantum transport, in which the conductance is given by the Landauer formula ${ }^{167-169}$

$$
G=\frac{2 e^{2}}{h} M T,
$$

where $-e<0$ is the fundamental electronic charge, $h$ is the Planck constant, $M$ is the number of transverse modes, and $T=|t|^{2}$ is the quantum transmission with $t$ (complex) being the transmission amplitude. Due to quantum interference, it is typical for the quantum transmission (and hence the conductance) to depend sensitively on transport parameters such as the conducting energy of the electron-the Fermi energy, the strength of the external magnetic field (if there is one), the confinement potential, or some other parameters, leading to conductance fluctuations.

Conductance fluctuations are in fact a fundamental phenomenon in open quantum systems. An important result is the universal conductance fluctuations (UCFs) in mesoscopic systems. ${ }^{170-174}$ In recent years, there have been studies of conductance fluctuations in graphene systems. ${ }^{132,137,138,175-181}$ The seminal work of Jalabert, Baranger, and Stone ${ }^{55}$ suggested that conductance fluctuations in the ballistic regime can be a probe of quantum chaos, establishing for the first time a connection between quantum transport in solid-state devices and classical chaos. Subsequent studies ${ }^{56-59,62-65}$ revealed that UCFs are intimately related to quantum chaotic scattering. ${ }^{53,54,57}$ An intriguing phenomenon is that classical chaos can suppress the conductance (or transmission) fluctuations. ${ }^{128,131,139}$ As shown in Fig. 3, for the two cases where classically the device geometry generates regular and chaotic dynamics, respectively, the transmission fluctuations are more severe in the former than in the latter. The physical origin of this striking contrast lies in the high probability for Fano resonances ${ }^{182-186}$ to arise in the case of classically regular dynamics. In particular, there are stable periodic orbits associated with regular classical dynamics. In the ideal case of a perfect lattice and zero temperature, a classical stable orbit has an infinite lifetime. In a realistic system with impurities at finite temperatures, the lifetime $\tau$ will not be infinite but can still be extremely long compared with the typical time scale of the system (e.g., the average time for an electron to drift through the device). Such "metastable" states, also called pointer states, ${ }^{45,187-192}$ will lead to narrow resonances (Fano resonances) in the curve of quantum transmission versus Fermi energy, where the width of the resonance is approximately $\hbar / \tau$. For a different classically stable orbit, the resonance energy (or frequency) will differ. A number of classically stable periodic orbits will then lead to sharp resonances in the transmission curve. In contrast, for classically chaotic dynamics, there are no stable periodic orbits. While a weaker type of pointer states, i.e., the remnants of quantum scars, still exist, their lifetimes are typically not comparable with those associated with the stable orbits for regular classical dynamics, broadening the corresponding resonances. As a result, the transmission fluctuation curve appears smoother, as illustrated in Fig. 3. Figure 4 shows the contrast between the pointer 

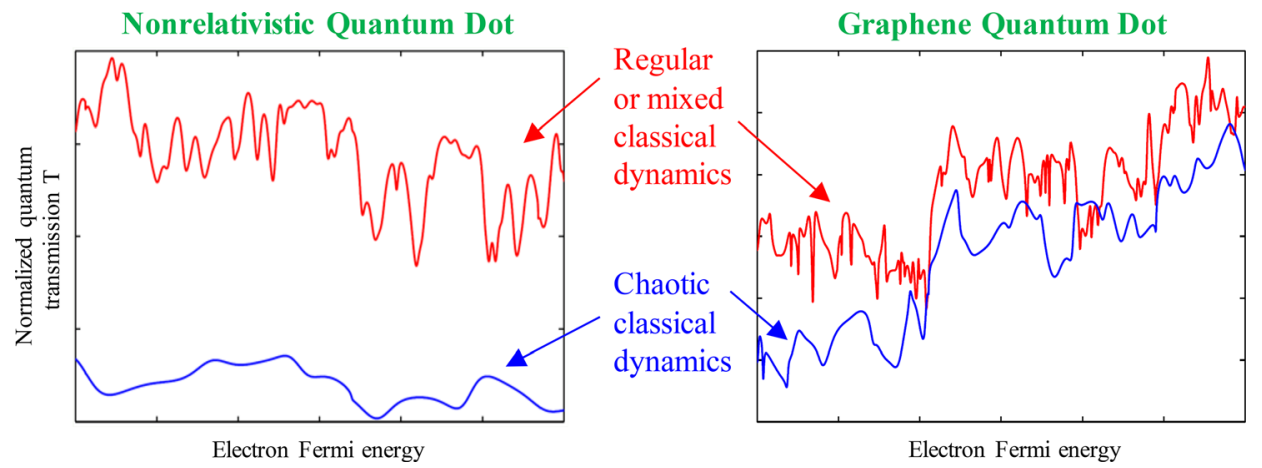

FIG. 3. Conductance fluctuations associated with transport through quantum dots: chaotic versus mixed dynamics, and nonrelativistic versus relativistic quantum transport. The left panel is conductance fluctuations for Schrödinger quantum dots (Fig. 4), while the right panel is for graphene quantum dots (Fig. 5). In both panels, the upper (red) and lower (blue) curves are for systems with mixed and chaotic classical dynamics, respectively. Chaos makes the fluctuation curves markedly smoother due to the much smaller probability to form Fano resonances of narrow width. For either mixed or chaotic dynamics, identical quantum dots are used here for the nonrelativistic (Schrödinger) and relativistic (graphene) quantum cases, so the differences in the conductance fluctuation patterns are due to the relativistic behavior of the quasiparticles.

states from a classically chaotic quantum dot structure (the panels to the left of the vertical green line) and those from a structure with mixed classical dynamics (on the right side of the green line), where it can be seen that the pointer states from the latter structure are more pronounced. ${ }^{128}$ A similar contrast exists in the relativistic quantum realm, as illustrated in Fig. 5 for the same geometries as in Fig. 4, where the quantum dot is made of graphene. ${ }^{128,191}$ These results established that, relativistic quantum transport through single-layer graphene systems, for which the quasiparticles are massless Dirac fermions, obeys the conventional classical-quantum correspondence that integrable or mixed classical dynamics lead to sharp conductance fluctuations but chaos can smooth out the fluctuations. However, under the same chaotic geometry, a relativistic quantum system (e.g., a graphene quantum dot) tends to exhibit more severe conductance fluctuations than its nonrelativistic counterpart, as illustrated in the right panel of
Fig. 3. Comparing the left-side panels of Figs. 4 and 5, we see that the pointer states in the graphene dot are more pronounced even when the classical dynamics are fully chaotic, pointing to a characteristic difference between relativistic and nonrelativistic quantum manifestations of the same classical dynamics.

An open issue concerns about the effect of finite mass on relativistic quantum transport. This can be addressed by studying quantum transport in chaotic bilayer graphene quantum dots for which the quasiparticles have a finite mass. A recent work $^{181}$ revealed that such a quasiparticle, when traveling along the classical ballistic orbit, tends to hop back and forth between the two layers, exhibiting a Zitterbewegung-like effect. Signatures of sharp conductance variations were uncovered, indicating that the effects of the finite mass in bilayer graphene systems are not significant enough to eliminate the sharp conductance fluctuations.

\section{Classical chaotic dynamics}


\section{Classical mixed dynamics}
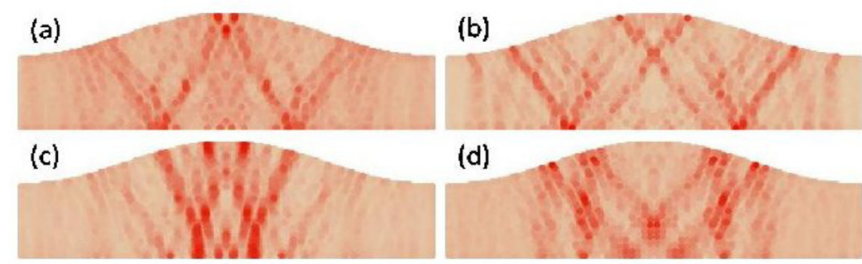

(d)

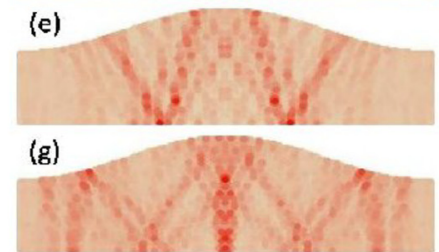

(h)
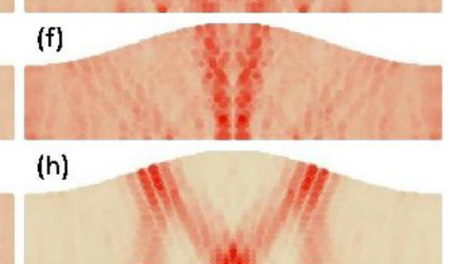

FIG. 4. Contrast in pointer states from classically fully chaotic and mixed quantum dot structures. The quantum dot has the structure of a cosine billiard, ${ }^{62}$ defined by two hard walls at $y=0$ and $y(x)=W+(M / 2)[1-2 \cos (2 \pi x / L)]>0$, respectively, for $0 \leq x \leq L$, where two semi-infinite leads of width $W$ are attached to the left and right openings of the billiard. By adjusting the ratios $W / L$ and $M / L$, the stabilities of the classical periodic orbits can be altered, allowing the transition from mixed to fully chaotic scattering. The panels to the left of the vertical green line are for the case where the classical scattering dynamics are fully chaotic (for $W / L=0.36$ and $M / L=0.22$ ) for four different values of the Fermi energy, while the panels on the right side of the green line correspond to mixed classical dynamics where there are stable periodic orbits (for $W / L=0.18$ and $M / L=0.11$ ) for eight Fermi energy values. The states are nonrelativistically quantum for which the Schrödinger equation was used to calculate the density of states through the standard method of nonequilibrium Green's function. ${ }^{128}$ It is apparent that the pointer states for the case of mixed classical dynamics are more pronounced. 


\section{Classical chaotic dynamics}
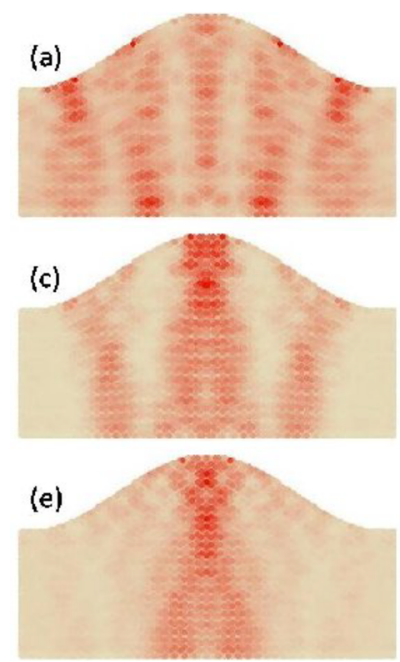


\section{Classical mixed dynamics}

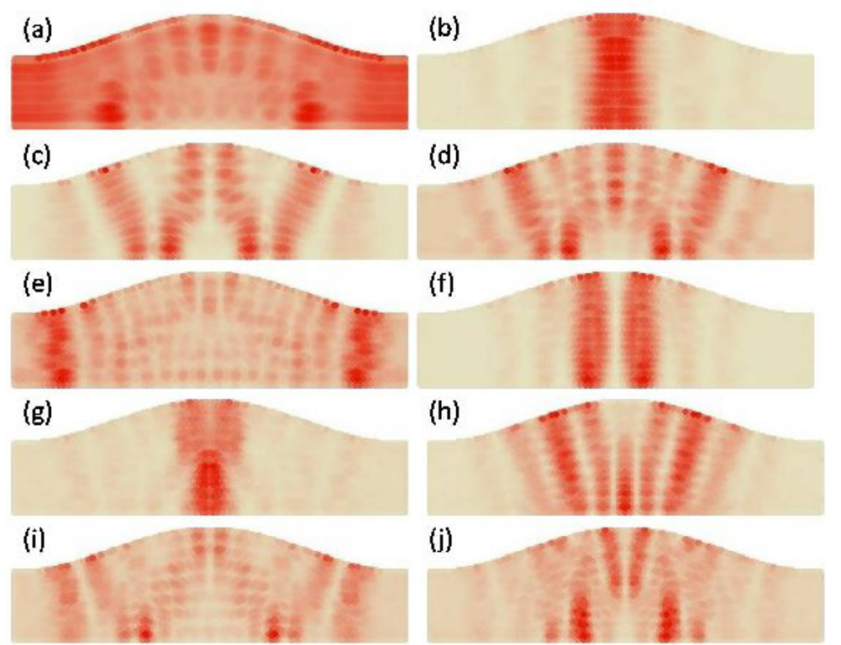

FIG. 5. Contrast in pointer states in classically fully chaotic and mixed graphene quantum dot structures for different values of the Fermi energy. The geometric structures are identical to those in Fig. 4, but the quantum dots are made of graphene. As in Fig. 4, the dot exhibiting mixed classical dynamics generates more pronounced quantum pointer states. Comparing the left patterns with those in the left side of Fig. 4, we see that even when the classical dynamics are fully chaotic, the pointer states in the graphene dot are more pronounced than those in the Schrödinger dot, leading to more severe conductance fluctuations in the former. This characteristic has a relativistic quantum origin and is different from the nonrelativistic counterpart under identical classical chaos.

Nevertheless, in cases where the mass contribution to the total energy is dominant, the system returns to being nonrelativistic quantum, where sharp conductance fluctuations in the chaotic case are removed. In solid-state electronic devices made of Dirac materials, sharp conductance fluctuations are thus expected, regardless of whether the quasiparticles are massless or have a finite mass.

Quite recently, a remarkable quantum scattering phenomenon in two-dimensional Dirac material systems was uncovered, ${ }^{193}$ where the manifestations of both classically integrable and chaotic dynamics emerge simultaneously and are electrically controllable. In particular, in analogy to a dielectric annular cavity, one can consider an electrical gate potential defined junction system with a ring geometry for massless Dirac fermions. Classically, this system generates a spectrum of dynamics (integrable, mixed, or chaotic), depending on the ring eccentricity and the effective refractive index configuration. The index can be electrically tuned to negative values to enable Klein tunneling. Inside the gated region, one can use ferromagnetism to lift the electron spin degeneracy through an exchange field. This leads to a class of spin-resolved, electrically tunable quantum systems of electron optics with massless Dirac fermions. With a proper gate potential, the spin-dependent refractive index profile can be controlled to generate regular ray dynamics for one spin state but generically irregular behavior with chaos for the other. A number of highly unusual physical phenomena arise, such as enhanced spin polarization with chaos, simultaneous quasi-scarred and whispering gallery type of resonances, and spin-selective lensing with a starkly near-field separation between the local density of states for spin up and down particles, which have application values in the development of 2D Dirac materials based electronic and spintronic devices.

\section{REGULARIZATION OF RELATIVISTIC QUANTUM TUNNELING BY CHAOS}

The phenomenon of regularization of quantum resonant tunneling by classical chaos was first uncovered by Pecora et al. ${ }^{194,195}$ Resonant tunneling is the basic physical principle underlying electronic devices such as resonant diodes. Such a system is basically a closed, (often) symmetric cavity, where a finite potential barrier is placed along the line of symmetry. The barrier separates the whole cavity into two symmetric domains: one on the left and another on the right side of the symmetric line. An electron can stay in either domain for a finite time before tunneling to the other, forming a resonant state, where the tunneling rate is inversely proportional to the average dwelling time of the electron in either domain (in the classical sense). For simplicity, we focus on two dimensions. The shape of the whole cavity can be as simple as a rectangle, where the classical dynamics are integrable. Or it can have a bowtie shape where three of the four boundaries have a constant negative curvature with the fourth one (the bottom boundary) being a straight line segment, so the classical dynamics are fully chaotic. Quantum mechanically, because the whole system is closed, there are an infinite number of eigenenergies - any eigenstate can be a resonant state with a tunneling rate. In a pertinent energy interval, there can then be a number of eigenstates with distinct energy values. If the classical dynamics are integrable, the values of the tunneling rate for these energy values can be distributed in a wide range or have a large spread. The remarkable phenomenon is that, when the classical dynamics are fully chaotic, the spread in the tunneling rate is greatly suppressed, henceforth the term regularization of quantum tunneling. ${ }^{194,195}$

In Refs. 194 and 195, the systems studied were nonrelativistic quantum mechanical. The question of whether 
chaos regularized resonant tunneling can occur in relativistic quantum systems was subsequently addressed ${ }^{133,141}$ using graphene and Dirac fermion systems. A somewhat subtle issue is the definition and calculation of the tunneling rate. In a nonrelativistic quantum system governed by the Schrödinger equation, the solution is straightforward as the tunneling rate can be found by identifying a pair of closely related eigenstates: one symmetric and another antisymmetric, whose energy difference $\Delta E$ essentially gives the rate. This can be seen from Fig. 6, where the top two panels show such a pair of eigenstates, denoted as $\Psi_{S}$ and $\Psi_{A}$, respectively. In the domain to the left of the potential barrier, the parts of $\Psi_{S}$ and $\Psi_{A}$ are perfectly identical, whereas in the right domain, they have opposite signs. The linear superpositions $\Psi_{S} \pm \Psi_{A}$ thus give a wave packet completely localized in the left half or right half of the cavity, respectively, as shown in the lower panels of Fig. 6. The time evolution between the superposition states $\Psi_{S} \pm \Psi_{A}$ is determined by the exponential factor $\exp (i \Delta E t / \hbar)$, so the tunneling rate is simply proportional to $\Delta E$.

While the symmetric and antisymmetric eigenstates can be readily found in the Schrödinger system due to the Dirichlet boundary condition where the wavefunctions assume zero value at the boundary, for the Dirac equation, the boundary conditions are of the zero normal flux type (see the Appendix). In this case, it is no longer feasible ${ }^{133,141}$ to identify a large number of symmetric pairs of spinors and to calculate $\Delta E$. A practical and computationally efficient method for calculating the resonant tunneling rate was then proposed, ${ }^{133,141}$ which is generally applicable to any quantum system, whether it be a lattice system, be governed by the Dirac or the Schrödinger equation. As shown in Fig. 7, for a resonant tunneling system of arbitrary domain shape, one first removes the tunneling potential barrier and calculates a large number of eigenstates. For any such state $\Psi_{n}$ with a definite energy value, one artificially truncates the wavefunction (or the spinor) by removing a significant portion of the component in the right half so that most of the state concentrates on the left half, as shown in Fig. 7(a). One then imposes the potential barrier along the symmetric line of the system, evolves the truncated state in time using the underlying equation (Dirac or Schrödinger), and calculates the probability $P_{L}$ that the state is in the left half of the system. Figure 7(b) shows an example of the time evolution of the probability $P_{L}$ for a Dirac fermion system, where initially about $90 \%$ of the magnitude sum of the two spinor components is concentrated in the left half. After a time $\Delta T$, the probability $P_{L}$ reaches its first minimum, with a reduction $\Delta P$ in its value. This means that, at $t=\Delta T$, the spinor state initially localized in the left half has largely tunneled through the barrier and is now mostly localized in the right half. The tunneling rate can thus be defined as

$$
\text { Resonant Tunneling Rate }=\pi \frac{\Delta P}{\Delta T} .
$$

The validity of this empirical but general and practically useful definition of the quantum resonant tunneling rate was established by the analysis ${ }^{133,141}$ that, for the nonrelativistic quantum system, it reduces exactly to one given by the energy difference $\Delta E$.

Figures 8(a) and 8(b) demonstrate the remarkable phenomenon of regularization of relativistic quantum resonant tunneling by classical chaos, ${ }^{133,141}$ where (a) and (b) are for a graphene and a Dirac fermion resonant cavity, respectively. The left columns in (a) and (b) show the tunneling rate versus the resonant energy for the two types of geometric domains in which the classical dynamics are integrable (rectangle, upper) and chaotic (bowtie, lower). For the rectangular domain, there is a significant spread of the tunneling rate for both the graphene and Dirac fermion systems, whereas this spread is greatly suppressed for the bowtie domain in which the

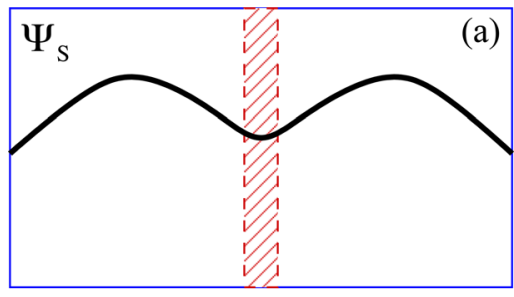

Symmetric pair

$\Psi_{\mathrm{S}}+\Psi_{\mathrm{A}}$
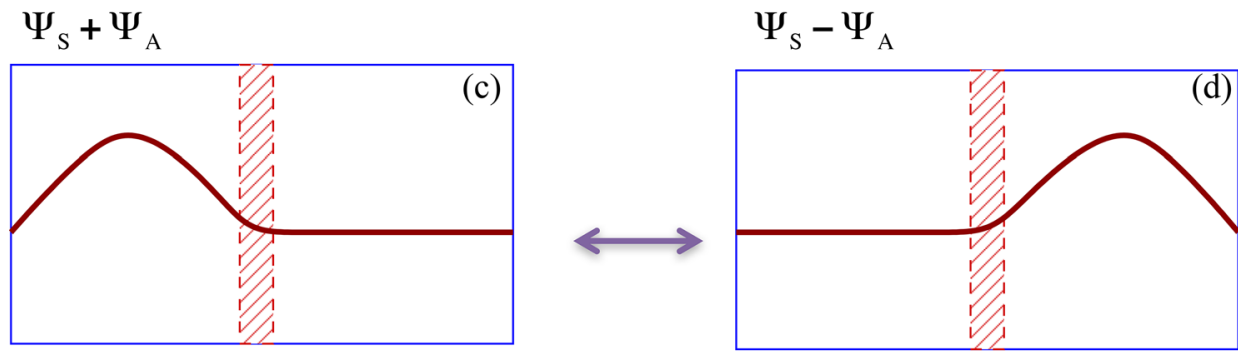

FIG. 6. Calculation of the resonant tunneling rate in nonrelativistic quantum systems. For systems governed by the Schrödinger equation, because of the Dirichlet boundary condition, it is natural and straightforward to find a pair of "kin" wavefunctions: one symmetric and another antisymmetric, as in (a) and (b), respectively. The symmetric and antisymmetric superpositions of these two wavefunctions then give a wave packet perfectly localized in the left or right domain, respectively, as in (c) and (d). The quantum time evolution of the superposition states stipulates that the resonant tunneling rate be proportional to the energy difference between the original wavefunction pair in (a) and (b).

In nonrelativistic quantum tunneling $\Delta \mathrm{t}=\frac{\pi \hbar}{\Delta \mathrm{E}} \Rightarrow$ tunneling rate $\propto \Delta \mathrm{E}$ 



Tunneling Rate $=\pi \frac{\Delta \mathrm{P}}{\Delta \mathrm{T}} \Rightarrow \Delta \mathrm{E}$ for nonrelativistic case

FIG. 7. A general and practical method to calculate the resonant tunneling rate for any quantum system. Given a symmetric resonant tunneling system, one first calculates a large number of eigenstates in the absence of the potential barrier. For such an eigenstate $\Psi_{n}$ associated with a definite eigenenergy [left side of panel (a)], one truncates its right component to obtain an artificial initial state $\Psi(t=0)$ [right side of panel (a)]. One then imposes the potential barrier, calculates the time evolution of the probability $P_{L}$ that the state is found in the left half of the system, and determines the time $\Delta T$ at which $P_{L}$ reaches its first minimum [panel (b)]. If the reduction in $P_{L}$ from $t=0$ to $t=\Delta T$ is $\Delta P$, the tunneling rate is defined as $\pi \Delta P / \Delta T$. In actual calculation, it is not necessary to have a truncated state that is localized completely in the left half [i.e., $P_{L}(t=0)=1$ ]. Insofar as most of the truncated state is in the left half and the reduction in its value when it reaches the first minimum is significant, the tunneling rate so defined is physically reasonable. In fact, for nonrelativistic quantum systems described by the Schrödinger equation, this definition of the tunneling rate reduces exactly to the one determined by the energy difference between a pair of symmetrically related eigenstates. ${ }^{133,141}$

classical dynamics are chaotic. That is, chaos effectively regularizes relativistic quantum tunneling, leading to a well defined tunneling rate for a small energy interval about any resonant energy value, which is desired from the standpoint of stable device operation. The right columns in (a) and (b) show some representative resonant eigenstates (for the energy value corresponding to the respective red dots in the left columns). For (a), the two panels show the density distributions on the two sublattices ( $A$ and $B$ ) of graphene, which correspond to the two pseudospin components. For (b), illustrated are the distributions of the two components of the spinor. For comparison, regularization of tunneling in the nonrelativistic counterpart system is shown in Fig. 8(c), where the eigenstate is a scalar wavefunction with one component.

While regularization of resonant tunneling by classical chaos is a phenomenon in both nonrelativistic and relativistic quantum systems, a feature that is uniquely relativistic quantum mechanical is the finite tunneling rate even when the resonant energy tends to zero. For nonrelativistic quantum tunneling systems, in the small energy regime, the potential barrier is effectively arbitrarily large so that the tunneling probability decays exponentially to zero, as can be seen from Fig. 8(c). However, in relativistic quantum systems, Klein tunneling can arise, where the tunneling probability can be one even if the potential barrier is wide and its height is much higher than the particle energy. As a result, in the near zero energy regime, there can be resonant states where tunneling occurs with a high probability, leading to an appreciable tunneling rate, which is particularly apparent for the Dirac fermion system, as shown in Fig. 8(b). For the graphene system, a signature of this manifestation of Klein tunneling can still be seen, as shown in Fig. 8(a), in spite of the absence of resonant states associated with near zero eigenenergies.

In graphene, Klein tunneling was observed quite early, ${ }^{196}$ which can have a significant effect on the transport behaviors. For example, it was predicted theoretically that Klein tunneling can lead to an extreme type of conductance

\section{(a) Graphene}

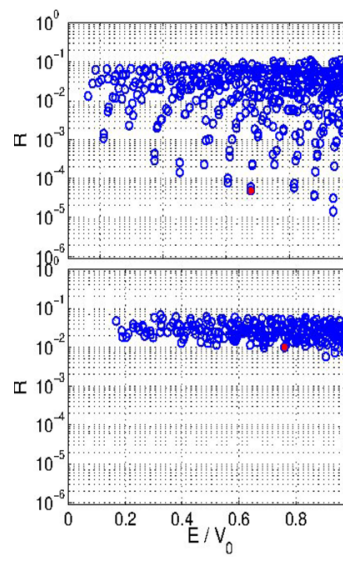

(b) Dirac fermion

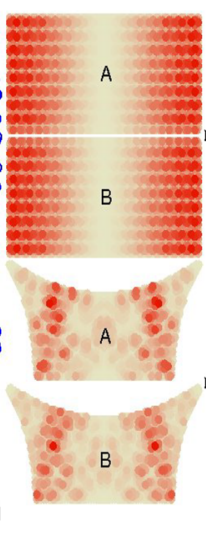

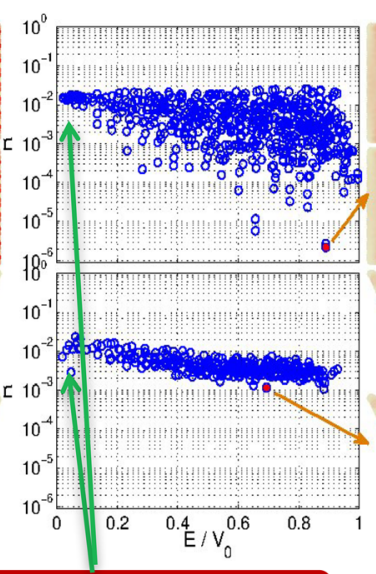

\section{(c) Schrodinger particle}

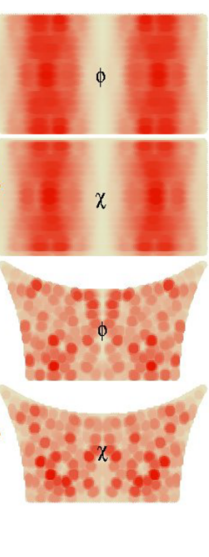

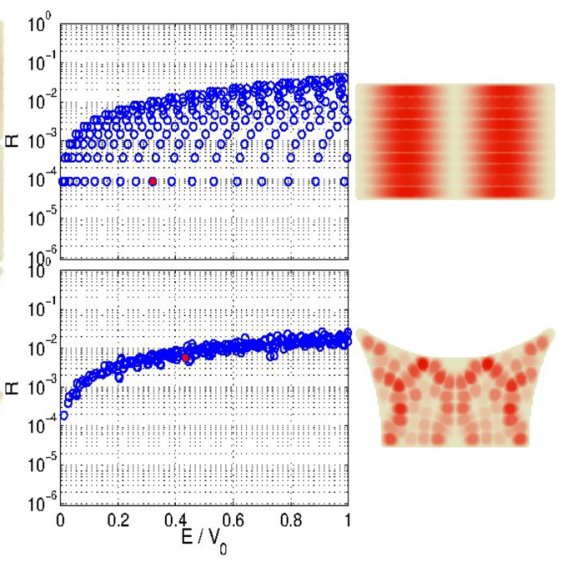

Fingerprint of Klein tunneling

FIG. 8. Regularization of relativistic quantum resonant tunneling by classical chaos. (a) For a graphene device, the tunneling rate versus the resonant energy for the two cases where the classical dynamics are integrable (rectangular cavity-upper panel) and chaotic (bowtie cavity, lower panel). For the rectangular cavity, there is a significant spread in the tunneling rate, which is markedly suppressed in the bowtie cavity. The right column in (a) illustrates representative resonant states (corresponding to the respective red dots in the left column), where "A" and "B" stand for the pseudospin states associated with the two sublattices of graphene. (b) Results for a Dirac fermion device, with similar legends to those in (a), except that, for the right column, the distributions shown are for the two spinor components. (c) The corresponding behavior in the nonrelativistic quantum device with a scalar wavefunction (henceforth only one state panel). A key difference between nonrelativistic and relativistic quantum resonant tunneling lies in the behavior of the tunneling rate near zero energy, where this rate tends to zero for the former (c) and remains appreciable for the latter (b). This is due to the uniquely relativistic quantum phenomenon of Klein tunneling where a particle can tunnel through a higher potential barrier with probability one, which absolutely has no counterpart in nonrelativistic quantum mechanics. 
fluctuations for transport through a quantum point contact (QPC). ${ }^{154}$ Specifically, a QPC can be modeled as a local gate potential applied to a graphene stripe. A calculation of the conductance versus, e.g., the relative potential height, reveals the existence of backscattering-zero-conductance dips caused by zero-conductance Fano resonances. ${ }^{197}$ However, due to Klein tunneling, interspersed with the dips are parameter values for which the conductances are large. As a result, a fractal-like type of conductance fluctuation pattern arises: the conductance can exhibit finite and zero values in a highly intermittent manner ${ }^{154}$ over distinct scales, as shown in the inset of Fig. 9. Mapping out representative electronic paths through a detailed calculation of the local current density revealed that, corresponding to the high conductance values, the electron tends to choose paths that pass through potential barriers of height as large as twice the electron energy-a clear signature of Klein tunneling, as shown in Fig. 9. The fractal-like behavior may pose a challenge on the functional stability of devices of Dirac materials.

\section{PERSISTENT CURRENTS IN CHAOTIC DIRAC FERMION SYSTEMS}

A remarkable phenomenon in quantum systems is persistent or permanent currents that require no external voltage, which can circulate with zero resistance. The traditional setting for persistent currents to arise is superconductors. However, in the early eighties of the last century, it was conceived theoretically ${ }^{198}$ that the dissipationless currents can arise from nonrelativistic quantum systems such as a normal metallic or a semiconductor ring, insofar as there is an
Aharonov-Bohm $(\mathrm{AB})^{199}$ magnetic flux through the center of the ring. Intuitively, this can be understood, as follows. For a metal ring of sufficiently small size, e.g., size less than the smaller of the phase coherence and elastic scattering lengths, the electron motions in the ring domain are effectively ballistic without any scattering. In the absence of any magnetic field, time reversal symmetry renders equal the magnitude of the currents in the clockwise and counterclockwise directions, so they cancel exactly, leading to a zero net current. However, a magnetic field or even a highly localized magnetic flux through the ring center breaks the time reversal symmetry. As a result, a net current can arise as soon as the magnetic flux is turned on, which does not require any external voltage to drive the current. To observe persistent currents, not only is it necessary for the ring size to be small, but the environmental temperature must be low in order for inelastic scattering from phonon-electron and/or electronelectron interactions to be insignificant. ${ }^{198,200-202}$ Another requirement is that the electron density should be sufficiently low so that electron-electron interactions can be neglected. In spite of the low temperature requirement, the metallic or semiconductor material itself is not superconducting and remains to be "normal." Experimental observations of persistent currents have been achieved for a variety of nonrelativistic quantum material systems. ${ }^{203-210}$

There is a correspondence between elastic scattering from randomly distributed impurities in the domain and that from deformed ("chaotic") boundaries. It is known that random impurities in one-dimensional or two-dimensional, metallic or semiconductor systems, can diminish the persistent

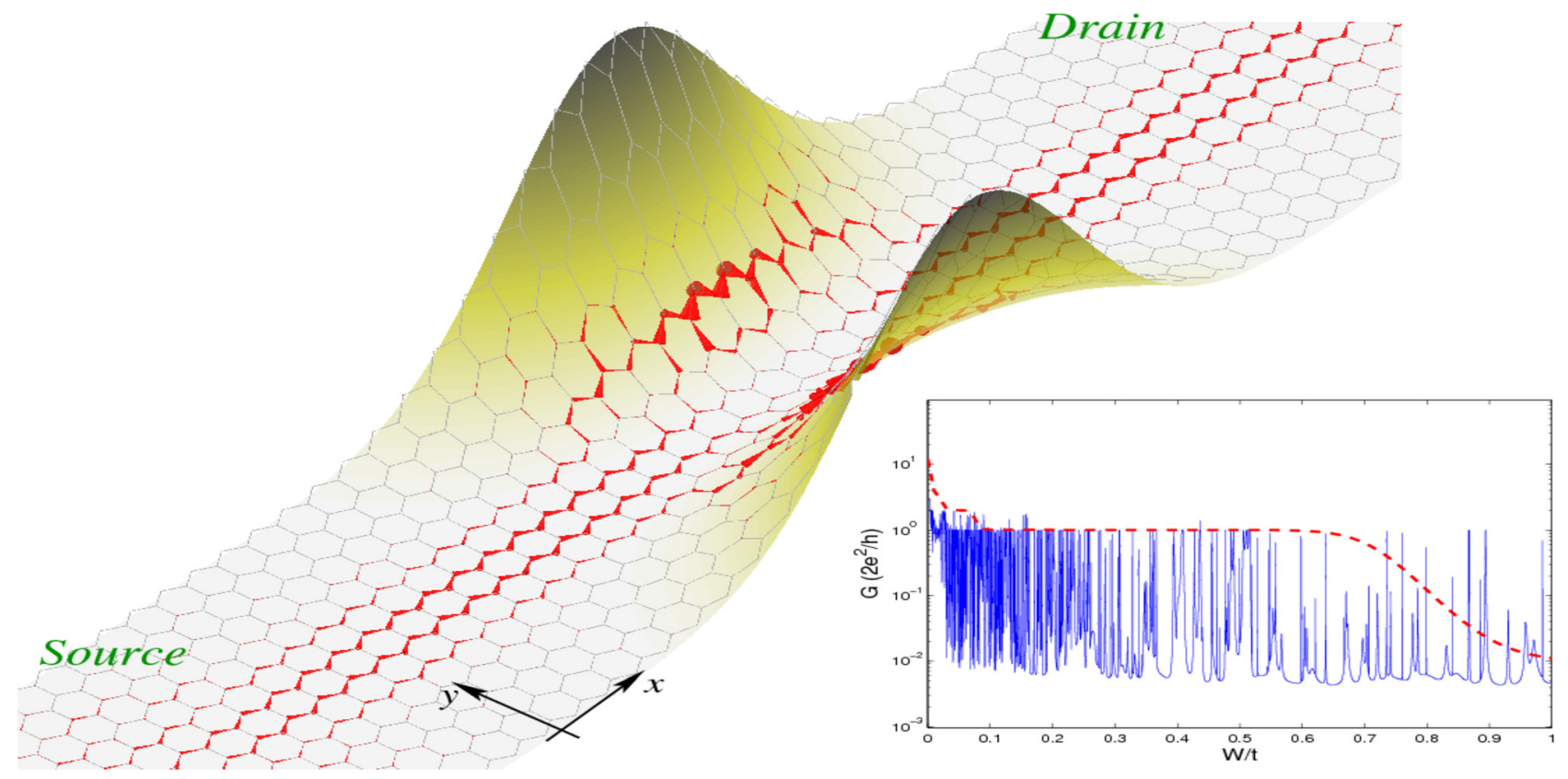

FIG. 9. Manifestation of Klein tunneling in a graphene quantum point contact: a fractal-like type of conductance fluctuations. The quantum point contact was modeled by a local gate potential of the form $V(x, y)=W \exp \left(-x^{2} / \xi^{2}\right) y^{2}$, applied to a graphene nanoribbon, where the parameter $W$ measures the tightness of the potential and $\xi=10 a$, with $a$ being the graphene lattice constant. The inset shows the normalized conductance through the point contact versus $W / t$, where $t$ is the nearest neighbor hopping energy of graphene, which exhibits an extreme type of, fractal-like behavior. The zero conductance dips correspond to backscattering Fano resonances, while the high conductance values are the result of Klein tunneling. The red dashed line represents the smooth conductance variation in the corresponding nonrelativistic quantum point contact. Some electron paths are shown by the connected red arrows that represent the local current flows, which go through potential regions of height that can be twice as large as the electron energy. The features here have no counterparts in nonrelativistic quantum point contacts. 
currents, ${ }^{211-217}$ where they decay exponentially to zero as the strength of the disorder is increased. ${ }^{211,217}$ The physical reason is that random scattering from impurities can drastically reduce the phase coherent length, rendering persistent currents realizable only for systems at or below the mesoscopic scale. Boundary deformation can also hinder the emergence of persistent currents. Especially, when the ring domain is not perfectly circular, scattering from the boundary can be irregular. In terms of classical scattering, even smooth deformation of the boundary from that of a circle can result in chaos, rendering scattering from the boundary effectively random. Quantum mechanically, breaking from the circular symmetry can remove the energy degeneracies, induce level repulsion, and flatten the Aharonov-Bohm oscillations so as to suppress the circulating currents. Random scattering from a deformed boundary is thus equivalent to scattering from impurities, leading to wavefunction localization and zero current.

Studies of persistent currents in graphene $e^{218-228}$ and other Dirac materials ${ }^{229,230}$ suggested that the currents are quite robust in relativistic quantum material systems. An idea to place this on a firm ground is to take advantage of chaos to investigate the robustness of persistent currents in a class of deformed, chaotic Dirac ring domains. ${ }^{146,148}$ The domains are chosen so that they can be transformed into a circular ring region through a conformal mapping. ${ }^{136}$ This way the eigenenergies and eigenstates of the Dirac equation incorporating the vector potential of the external magnetic flux can be calculated with high accuracy. ${ }^{146}$ A remarkable result was that, even with severe deformation so that the corresponding classical dynamics in the domain are fully chaotic, robust persistent currents can arise, henceforth the term "superpersistent" currents in Dirac fermion systems. ${ }^{146}$

Figure 10 demonstrates the sharp contrast between the behaviors of persistent currents in nonrelativistic quantum (Schrödinger) and relativistic quantum (Dirac) domains for the two cases where the classical dynamics are regular (integrable) and chaotic, respectively. The upper left panel shows a general chaotic ring domain with an $\mathrm{AB}$ flux through the center (the small black dot). Two specific domains, one regular and another chaotic, are shown above panels (a) and (b), respectively. Figures 10(a) and 10(b) show the persistent current summing over the five lowest eigenstates versus the normalized magnetic flux parameter $\alpha$, for the Schrödinger domain, and the corresponding results for the Dirac domain are shown in Figs. 10(c) and 10 (d). It can be seen that, for the circular domain, there is a persistent current in both cases. However, for the chaotic domain, the current magnitude diminishes for the Schrödinger system but sustains relatively large values for the Dirac fermion system, indicating that for the latter, the current is superpersistent.

In the Schrödinger domain, classical chaos caused by random reflection from the deformed boundary leads quantum mechanically to localization of the eigenstates, as exemplified by Fig. 10(e), which effectively prevents the occurrence of any appreciable circulating current. In contrast, in the Dirac domain, the eigenstates are not localized so that a circulating current can still form. In fact, physically, the superpersistent currents ${ }^{146,148}$ in the Dirac domain is due to the emergence of the unusually robust whispering gallery modes (WGMs) near the boundaries of the domain, as shown in Fig. 10(f). Such modes carry a large angular momentum, which were usually studied in optical microcavity systems such as microlasing devices ${ }^{231-234}$ and were found in nonrelativistic quantum electronic systems ${ }^{235}$ as well. Here, due to the zero normal flux boundary conditions required for the Dirac equation to have nontrivial, physically meaningful solutions, a distinct set of WGMs sticking to the edge can arise and are insensitive to boundary deformation. Figure $10(\mathrm{~g})$ shows the maximum persistent current versus the geometrical parameter $g$ of the domain, where $g=0,0<g<1$, and $g=1$ correspond to classically integrable, mixed, and fully chaotic dynamics, respectively. For the Schrödinger ring, as the ring becomes increasingly chaotic, persistent currents vanish for $g \gtrless 0.6$. However, for the Dirac ring, even when the domain is fully chaotic, there is still a significant persistent current.

Further support for the robustness of the persistent currents in 2D relativistic quantum systems was obtained recently through a systematic study of the effects of random disorders. ${ }^{236}$ In the study, the same geometric setting of an infinite mass confined Dirac ring domain with a vertical magnetic flux through the center was used. Uncorrelated disorders were assumed to exist throughout the domain, which were simulated using localized, uniformly distributed random electric potentials. The total number of disorders in the whole domain was chosen as a control parameter, and the Dirac equation was solved numerically to obtain the magnitudes of the persistent currents as a function of the control parameter. It was found ${ }^{236}$ that, as the number of the disorders is systematically increased, the average current decreases slowly initially and then plateaus at a finite nonzero value, indicating that the persistent currents are robust. Whispering gallery modes along the domain edges were again identified to be the physical mechanism responsible for the robust currents. In contrast, in the nonrelativistic counterpart system subject to the same disorder distribution, these modes are sensitive and fragile, leading to a rapid and exponential decay of the currents to zero. A physical theory based on a quasi one-dimensional approximation was developed to understand the strikingly contrasting behaviors of the currents in the Dirac and Schrödinger rings in the presence of random disorders. Experimentally, for a given material, neither the strength nor the density of the disorders can be readily adjusted, but the sample size can be controlled. Since, classically, under a vertical magnetic field an electron moves along a circular trajectory in the domain, for a larger ring sample with constant disorder density, the electron encounters more disorders/scattering events in one complete rotation. An implication of the extraordinary robustness of the persistent currents in the Dirac ring system is then that they can occur in realistic systems of large size.

Studying the effects of classical chaos on relativistic quantum states can thus lead to insights into the robustness of remarkable physical phenomena such as persistent currents in Dirac fermion systems. With respect to potential applications, recently, it was proposed that the ring structure 

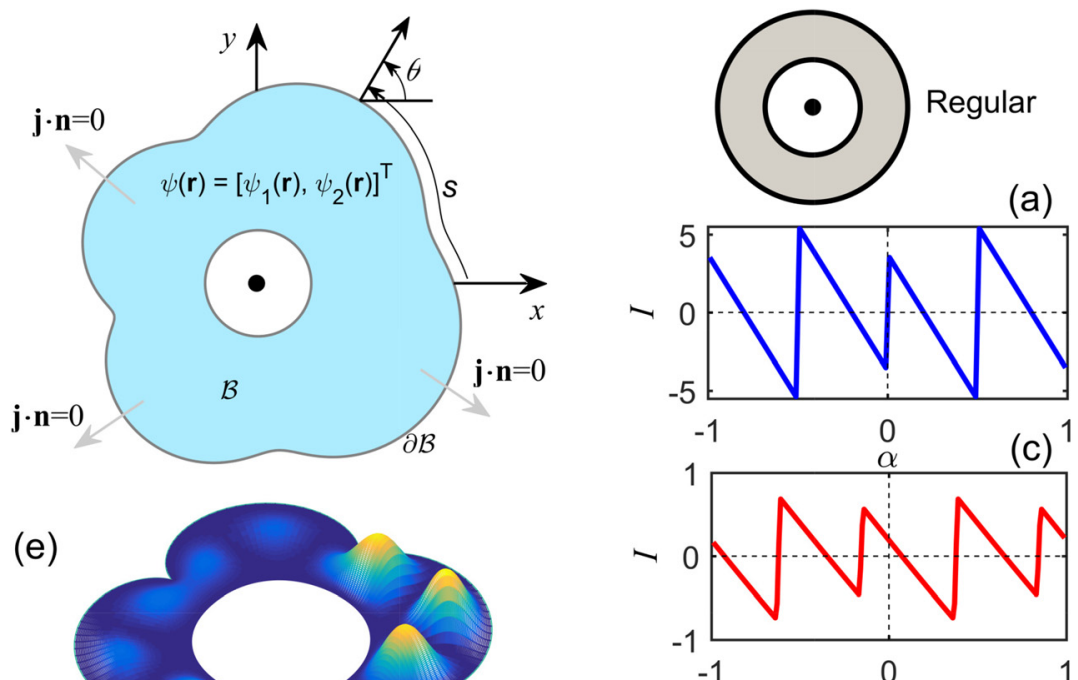

(c) ${ }^{1}$

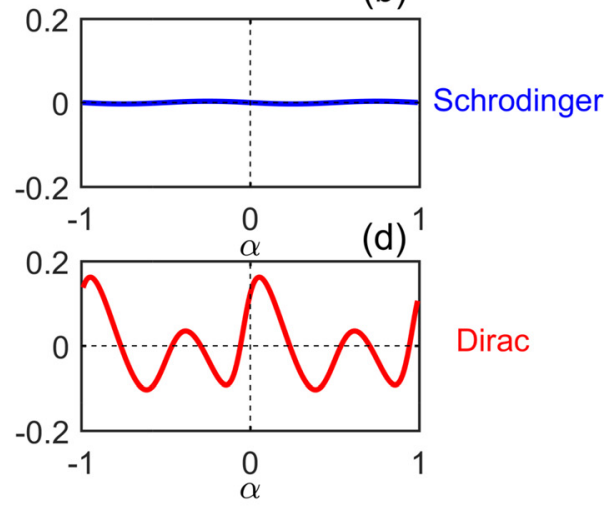

(d) 1

(b)

(e)

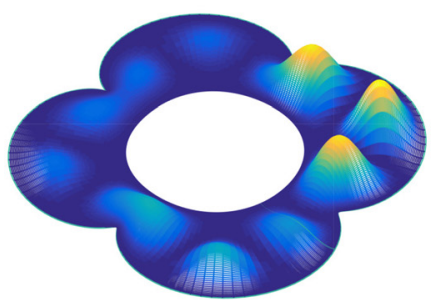

(f)
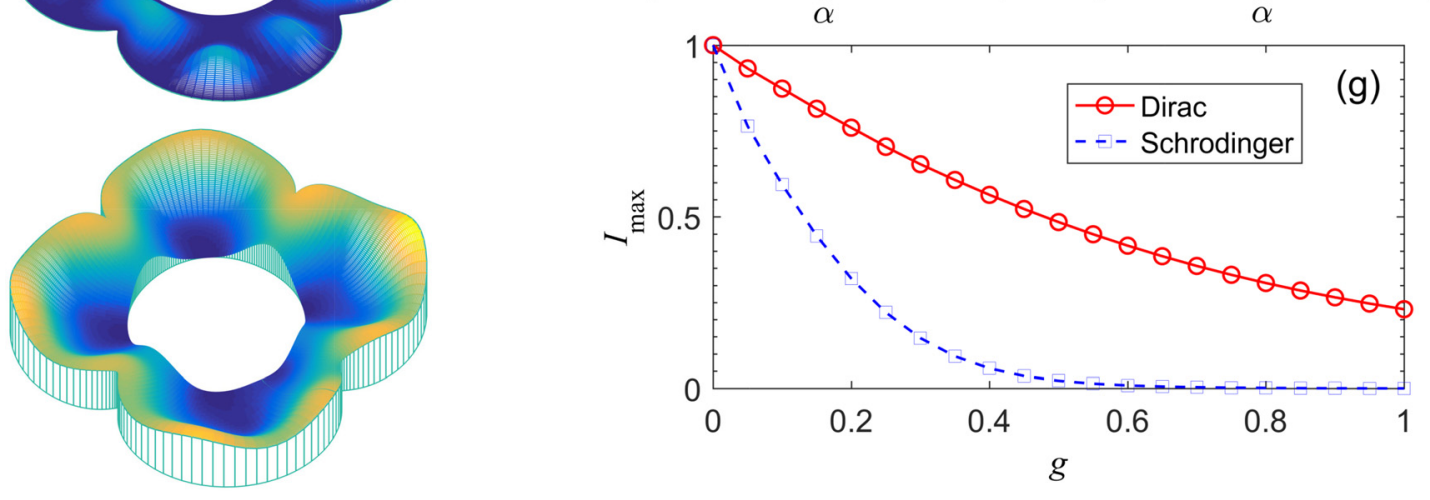

Dirac

FIG. 10. Superpersistent currents and whispering gallery modes (WGMs) in a chaotic Dirac domain. Upper left panel: a chaotic ring domain with an AB magnetic flux through the center of the ring. The boundary of the outer boundary is parametrized by the arc length $s$. For motion of a massless Dirac fermion inside the domain, the boundary condition is of the zero-flux type, i.e., no outward current at any point $s: \boldsymbol{j} \cdot \boldsymbol{n}=0$. Two geometrical domains are shown in the top row: one with regular dynamics and another generating chaos in the classical limit. The magnitude of the persistent current from the five lowest eigenstates versus the normalized magnetic flux: [(a) and (b)] Schrödinger ring and [(c) and (d)] Dirac ring. For the regular domain, there are persistent currents in both Schrödinger and Dirac systems, due partly to WGMs. Chaos diminishes the currents for the Schrödinger system (b), but they are sustained in the Dirac system regardless of chaos, giving rise to superpersistent currents (d). Physically, the destructive role of chaos in suppressing persistent currents in the Schrödinger system can be attributed to localization of the eigenstates due to random scattering from the deformed boundary (e). In contrast, in the Dirac system, along both the inner and outer boundaries, unusual WGMs "sneaking" through the deformation arise, leading to superpersistent currents, as exemplified by the patterns in (f). Panel (g) shows the maximum persistent current versus the geometrical parameter $g$ of the domain. In particular, the domain is generated by deforming the circular ring defined as $r_{1}=0.5 \leq r \leq 1.0=r_{2}$ in the $z$ plane through the conformal mapping $w(z)=h\left[z+0.05 g z^{2}+0.18 g\right.$ exp $\left.(i \omega) z^{5}\right]$, where $\omega=\pi / 2,0 \leq g \leq 1$ is the deformation parameter that controls the nature of the classical dynamics, and the normalization coefficient is given by $h=1 / \sqrt{1+\frac{g^{2}}{200}\left(1+r_{1}^{2}\right)+\frac{81 g^{2}}{500}\left(1+r_{1}^{2}+r_{1}^{4}+r_{1}^{6}+r_{1}^{8}\right)}$. When $g$ is increased from zero to one, the classical dynamics in the deformed ring domain will undergo a transition from being integrable $(g=0)$ to mixed $(0<g<1)$ and finally to being fully chaotic $(g=1)$. For the Schrödinger ring, as $g$ is increased from zero so that the ring becomes increasingly chaotic, persistent currents diminish for $g \gtrsim 0.6$. However, for the Dirac ring, even when the domain is fully chaotic, there are still significant persistent currents.

under a magnetic flux can serve as a robust relativistic quantum two-level system with edge-dependent currents and spin polarization. $^{148}$

WGMs have sizable contribution to the persistent currents, which is true for both relativistic and nonrelativistic quantum systems. However, WGMs are much, much more robust in the relativistic than in the nonrelativistic quantum systems! The key result in Fig. 10 is that the WGMs are destroyed in the Schrödinger ring [Fig. 10(b)] by chaos, but they persist in the Dirac counterpart [Fig. 10(d)]. In fact, drastically contrasting behaviors occur in the values of the wavefunctions at the ring boundary: for the Schrödinger system, the wavefunctions are zero there, but the spinor wavefunctions can have local maximum values at the boundary for the Dirac ring system.

\section{ENERGY LEVEL STATISTICS IN RELATIVISTIC QUANTUM CHAOTIC SYSTEMS}

The energy-level spacing statistics in closed Hamiltonian systems are a classic topic in nonrelativistic quantum chaos. $^{3-19}$ What has been known so far is the following. For a classically integrable system, the energy level spacings obey the Poisson distribution. For a fully chaotic system with a time reversal symmetry, the level spacing statistics follow the Wigner distribution, the so-called Gaussian orthogonal ensemble (GOE) statistics, associated with which is the phenomenon of level repulsion. When the time reversal symmetry is broken, e.g., by an external magnetic field, Gaussian unitary ensemble (GUE) statistics for the energy level spacings arise. 
For a relativistic chaotic quantum billiard, the smoothed wave vector staircase function for positive eigenvalues is given by ${ }^{96}$

$$
\langle N(k)\rangle=A k^{2} / 4 \pi+C_{1}+\cdots,
$$

where $A$ is the area of the billiard and $C_{1}=-1 / 12$. For a chaotic graphene billiard, about a Dirac point the energy-momentum relation is $E=\hbar v_{F} k=\sqrt{3} t a k / 2$ with $v_{F}=\sqrt{3} t a / 2 \hbar$ being the Fermi velocity. For the $n$th energy level $E_{n}$, one has

$$
k_{n}=\frac{2}{\sqrt{3} a} \cdot \frac{E_{n}}{t} .
$$

Once the eigenenergy $E_{n}$ is determined, the corresponding wave vector $k_{n}$ can be obtained through the above relation. For the chaotic graphene Africa billiard, one has ${ }^{123,127}$

$$
\langle N(k)\rangle=A k^{2} / 2 \pi+C_{1}^{\prime},
$$

where $C_{1}$ is a fitting constant. Equation (5) differs from Eq. (4) in the leading "Weyl" term by a factor of 2 . The reason is that, for a single Dirac point, $\langle N(k)\rangle$ follows Eq. (4) but graphene has two nonequivalent Dirac points, so $\langle N(k)\rangle$ should be twice of that given by Eq. (4). As a result, the denominator becomes $2 \pi$ (instead of $4 \pi$ ). The fitting constant $C_{1}^{\prime}$ is due to the edge states on the segments of the zigzag boundaries of the graphene billiard where their energies are all about zero. For a zigzag ribbon, the edge states exist for $E<E_{c}=\hbar v_{F} / L=\sqrt{3} t a /(2 L)$, where $L$ is the width of the ribbon. ${ }^{239}$ If the size of the graphene billiard is about $100 a$, one has $E_{c} \simeq 0.01 t$. The edge states are localized on segments of the zigzag boundaries. These states are essentially degenerate states, contributing to an artificial bias in the spectral staircase function for small energy values. Therefore, a minimum value for $E_{n}$, e.g., $2 E_{c}=0.02 t$, can be set to remove the influence of the edge states. ${ }^{123,127}$

Because of the linear energy-momentum relation $E=\hbar v_{F} k$, the smoothed spectral staircase function is given by

$$
\langle N(E)\rangle=\frac{A E^{2}}{2 \pi \hbar^{2} v_{F}^{2}}+C_{2}=a_{0} E^{2}+C_{2},
$$

where $a_{0}=A /\left(2 \pi \hbar^{2} v_{F}^{2}\right)$ is the unfolding normalization parameter and $C_{2}$ is now zero after setting $2 E_{c}$ for the minimum value of $E_{n}$.

The first contribution to our understanding of the statistics of relativistic quantum energy level spacing was due to Berry and Mondragon ${ }^{96}$ who studied the energy level statistics for a class of two-dimensional chaotic (Africa) neutrino billiard governed by the massless Dirac equation. The time reversal symmetry of the system is broken due to the infinite-mass confinement potential. As a result, the level spacing statistics are GUE, as shown in the first column of Fig. 11.

Level spacing statistics have been studied using chaotic graphene billiards. ${ }^{123,127,240,241}$ A relatively early work $^{240}$ addressed the role of chaos in the statistics of graphene quantum dots. For large size (e.g., $>100 \mathrm{~nm}$ ), the dot behaves as a conventional single-electron transistor, exhibiting periodic Coulomb blockade peaks. For small size (e.g., $<100 \mathrm{~nm}$ ), the peaks become aperiodic, indicating the dominant effect of quantum confinement. It was found ${ }^{240}$ that random peak spacing and its statistics are well described by the theory of chaotic quantum billiards. Similar features were also observed in a tunable graphene single electron transistor. ${ }^{241}$

A systematic study of the effect of chaos on the level spacing statistics in graphene billiards was subsequently carried out ${ }^{123,127}$ using the Africa billiard ${ }^{7}$ and one eighth of the Sinai billiard, where the nearest neighbor interacting, tightbinding Hamiltonian was used. The boundary of the graphene Africa billiard is described by $x+i y=64(z$ $\left.+0.2 z^{2}+0.2 z^{3} e^{i \pi / 3}\right) a$ with $a=\sqrt{3} a_{0}=2.46 \AA$ and $z$ denoting the unit circle in the complex plane. To attain the short wavelength regime, a relatively large billiard size is necessary, e.g., with area larger than $900 \mathrm{~nm}^{2}$ containing over 35000 atoms.

For a chaotic graphene billiard, since the quasiparticles with energies close to a Dirac point are massless and have a pseudospin $1 / 2$, they follow the same Dirac equation used by Berry and Mondragon. ${ }^{96}$ An intuitive thinking may be that the statistics should also be GUE as in Berry and Mondragon's neutrino billiard. ${ }^{240,242}$ However, numerical calculations revealed a strong signature of GOE statistics, ${ }^{123,127,242}$ as shown in the second and third columns of Fig. 11. This unexpected behavior can be understood, as follows.

For the chaotic neutrino billiard, the time reversal symmetry is broken because of chirality. ${ }^{96}$ Due to the two nonequivalent Dirac points (valleys) in graphene, the symmetry corresponds to the symplectic symmetry, which is essentially the time reversal symmetry for a single valley. ${ }^{243}$ Thus, the breaking of the time reversal symmetry in the neutrino billiard does not imply breaking of the symmetry in graphene billiards. More specifically, in graphene, quasiparticles in the vicinity of a Dirac point obey the same Dirac equation as that for neutrino, but the confinement to realize the billiard plays a different role, where the abrupt edge termination couples the two valleys in the momentum space. As a result, the wavefunctions for quasiparticles with wave vectors near the two Dirac points are not separable, rendering invalid the description of the two-component spinor Dirac equation for the whole system. A full set of equations taking into account the two nonequivalent Dirac points and the boundary conditions are thus necessary to describe the motions of the relativistic quasiparticle in graphene. Typically, the time reversal symmetry is preserved, ${ }^{243}$ suggesting that the level spacing statistics belong to either the GOE or the GSE (Gaussian symplectic ensemble) class. The abrupt edge termination in a graphene billiard can be described by a step function in the form of an infinite potential at the edge. Since the range of the potential is short, the two valleys in the momentum space are coupled, breaking also the sublattice symmetry. Now that both the pseudospin valley and the sublattice symmetries are broken, Kramer's degeneracy and GSE statistics can be ruled out. ${ }^{244}$ The resulting levelspacing statistics are then of the GOE type. Similar effects were noticed by Robnik and Berry ${ }^{92}$ that, in certain cases, 

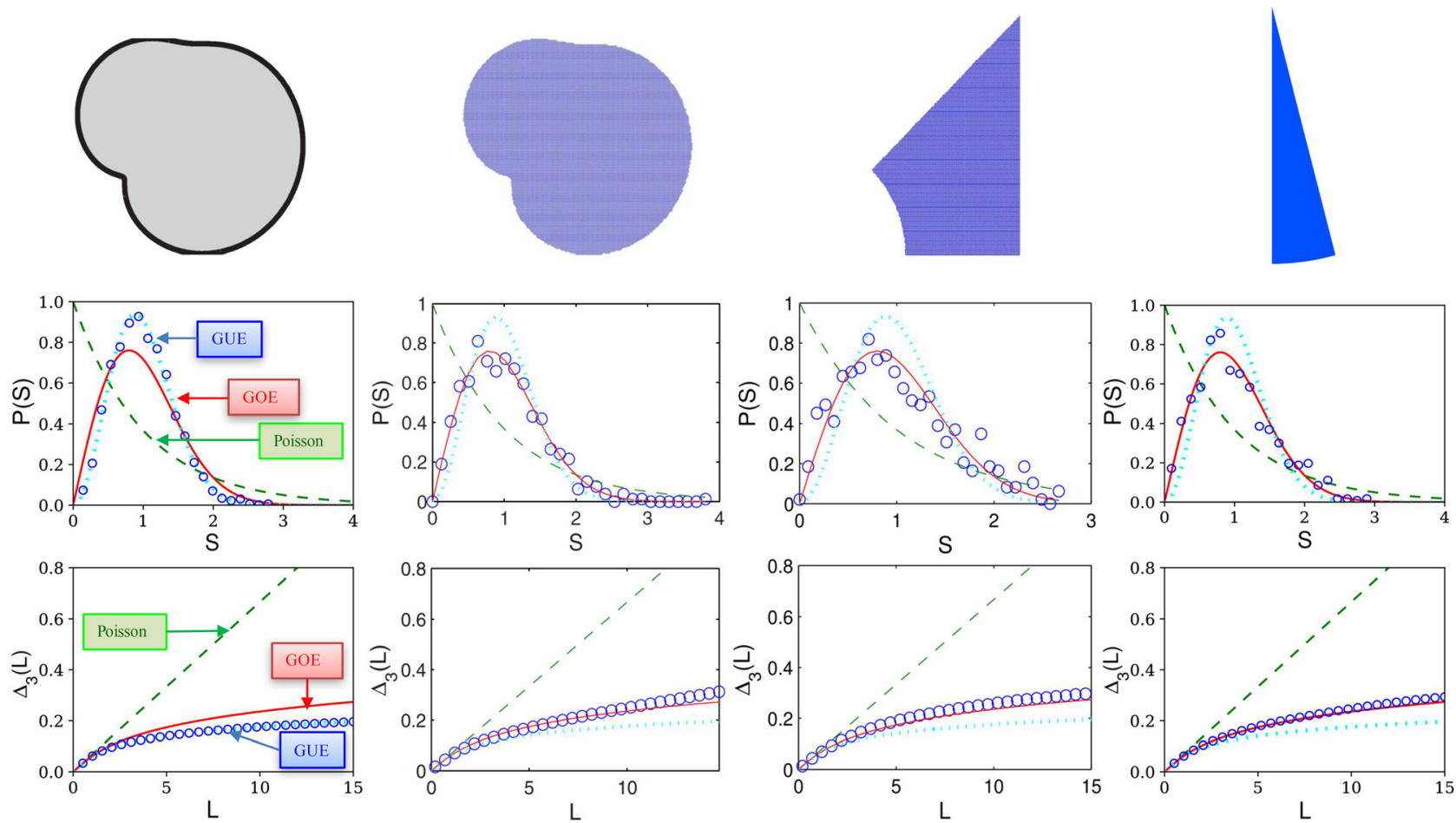

FIG. 11. Energy level spacing statistics of relativistic quantum billiards. The three rows show the geometric shapes of various billiards, the corresponding level spacing statistics $P(S)$, and the spectral rigidity $\Delta_{3}(L)$, respectively. The first column is for the neutrino billiard of Africa shape (fully chaotic), where the massless Dirac equation was solved using the conformal-mapping method ${ }^{136}$ with 20000 basis, and the first 4000 energy levels were used to calculate the level spacing statistics. ${ }^{96,237}$ Because of the breaking of the time reversal symmetry by the infinite mass confinement, the statistics are of the GUE type. The second column is for a graphene billiard of Africa shape (fully chaotic) with 42505 atoms, ${ }^{123}$ where 623 energy levels in the range of [0.02, 0.4] were used for the statistics. In this case, GOE statistics are observed. The third column is for the 1/8 of graphene Sinai billiard (fully chaotic) with 37401 atoms, and 549 energy levels in the range of $[0.02,0.4]$ were used for computing the level statistics. ${ }^{127}$ Again, the level spacing statistics are of the GOE type. The fourth column is for the $15^{\circ}$ graphene sector billiard (integrable) of 226993 atoms, and 837 energy levels in the range of [0.02, 0.2] were used for the statistics. Despite that the classical dynamics are integrable, the characteristics of GOE statistics emerge..$^{238}$

although the system possesses neither time-reversal symmetry nor geometric symmetry (or other dynamical symmetries), it can be invariant under the combination of the two symmetries, and nontrivial representations can be found in which the Hamiltonian matrix elements are real, leading to GOE but not to GUE statistics. Experiments using microwave photonic crystals to mimic graphene billiards ${ }^{245-249}$ have verified the GOE statistics for the chaotic Africa billiard system. ${ }^{248}$

When a magnetic field is present, the time reversal symmetry of graphene ${ }^{243}$ is broken, causing the level spacing distribution to exhibit characteristics of GUE statistics. This was numerically verified ${ }^{123,127}$ for both the Africa and oneeighth of the Sinai billiard subject to a uniform, perpendicular magnetic field.

An intriguing result for graphene billiards is that GOE statistics emerge even when the classical dynamics are generically integrable, as shown in the fourth column of Fig. 11. This is surprising because the Schrödinger equation for the sector billiard of any angle is analytically solvable, where the level spacing statistics are perfectly Poisson. A systematic investigation revealed that, the unexpected GOE behavior is due to the edge effects of the graphene system. ${ }^{238}$ In particular, for energies near the Dirac point where the quasiparticles behave like massless Dirac fermions, Poisson statistics are extremely rare in the sense that it emerges only under quite strict symmetry constraints on the straight boundary parts of the sector. An arbitrarily small amount of imperfection of the boundary will result in GOE statistics. An implication is that, for circular-sector confinements with an arbitrary angle, the spectral properties will generically be GOE, in spite of classical dynamics' being integrable.

We summarize this section by emphasizing that, in graphene systems, the dynamics are "relativistic quantum" only in a restricted sense: the Dirac equation applies near the Fermi level and within a single valley only. Boundary scattering and any other source of intervalley scattering spoil the analogy with relativistic particles. While topological insulators are a possible alternative platform, a confinement will necessarily induce time-reversal symmetry breaking. As pointed out by Berry and Mondragon, ${ }^{96}$ precisely because of the time reversal symmetry breaking are the energy level spacing statistics GUE. There are thus two types of systems with two characteristically distinct results: (a) the Dirac billiard corresponding to a confined domain of the Dirac equation or a chaotic confinement of topological insulator in which the time reversal symmetry is broken, leading to GUE statistics and (b) chaotic graphene billiards that preserve the time-reversal symmetry, where GOE statistics arise. Consequently, the GOE statistics examined in this Tutorial do not arise in chaotic confinement of topological insulators, provided that such a confinement does not possess a mirror symmetry or any other discrete symmetries. 


\section{DISCUSSION}

The tremendous recent advances in two-dimensional Dirac materials have raised the fundamental question about the manifestations of classical chaos in relativistic quantum systems, giving birth to the field of relativistic quantum chaos. This field is particularly relevant to developing novel solid state devices in electronics, spintronics, and even valleytronics. In this Tutorial, we discuss a number of topics in this emergent field. They are: relativistic quantum scars ${ }^{121,134,136}$ in Dirac fermion and graphene systems, peculiar features of relativistic quantum chaotic scattering and quantum transport, ${ }^{128,140,250}$ chaos based modulation of conductance fluctuations in quantum dot systems, ${ }^{131,139}$ regularization of relativistic resonant quantum tunneling by chaos, ${ }^{133,141}$ the manifestations of the Klein paradox in graphene or 2D Dirac fermion systems, ${ }^{154}$ superpersistent currents in chaotic Dirac rings subject to a magnetic flux, ${ }^{146,148}$ and energy level statistics in closed graphene and Dirac fermion systems that exhibit chaos in the classical limit. ${ }^{123,127,238}$ These results represent only initial, preliminary, and quite limited efforts in this field.

Research on relativistic quantum chaos so far has revealed a number of unexpected phenomena that are striking and counterintuitive with respect to traditional quantum chaos. These include chiral scars, sharp residual resonance associated with transport through chaotic graphene quantum dots, unconventional statistics of energy level spacing, manifestations of Klein tunneling, and an extraordinary degree of robustness of persistent currents in chaotic Dirac billiards. In fact, the specific orbital structure associated with chiral scars is uniquely a relativistic quantum manifestation of certain classical unstable periodic orbits, which find absolutely no counterparts in nonrelativistic quantum systems. The persistence of sharp resonances associated with conductance in chaotic graphene quantum dots is surprising resonances would be largely removed by chaos in the corresponding nonrelativistic quantum dot. While the study of energy level statistics is technically standard, that chaotic graphene billiards exhibits GOE statistics is surprising as the early work of Berry and Mondragon on chaotic neutrino billiards established GUE statistics due to breaking of the time reversal symmetry. Another unusual phenomenon is the occurrence of GOE statistics in graphene billiards even when the corresponding classical dynamics are integrable.

We wish to offer a special discussion of Klein tunneling, tunneling through a finite potential barrier with probability one, which is fundamentally a relativistic quantum phenomenon that is not possible in nonrelativistic quantum systems. It can occur in systems regardless of the nature of the classical dynamics, i.e., integrable or chaotic. In Sec. IV, we point out the signatures of Klein tunneling in Dirac fermion and graphene resonant-tunneling systems, which have been found for both integrable and chaotic classical dynamics. A previous work ${ }^{154}$ on graphene-based quantum point contacts also demonstrated that abnormal electron paths due to Klein tunneling can occur and lead to fractal-like conductance fluctuations. Since, in general, the occurrence of Klein tunneling requires certain specific incident angle of the particle, we can intuitively expect that chaos, due to the broad angle distribution associated with it, makes relativistic quantum confinement more difficult than integrable dynamics. However, at the present, the interplay between Klein tunneling and distinct types of classical dynamics has not been well understood.

A complete understanding of the various phenomena arising from the interplay between classical dynamics and relativistic quantum mechanics requires an analysis of the quantum-classical correspondence in the context of the Dirac quantum theory, which remains an outstanding topic. For example, chiral scars are due to the inherent chirality of the massless Dirac fermions and the subtle time-reversal symmetry breaking imposed by a massive type of hard wall confinement in reduced two spatial dimensions (without any external magnetic field). A broken time-reversal symmetry is responsible for parity anomaly - a striking phenomenon implied by the Dirac theory for fermions in $(2+1)$-dimensional space-time. That is, the fundamental parity symmetry associated with the classical action does not imply the same symmetry in the corresponding relativistic quantum system. (The subtle time-reversal anomaly can be due to a sustained Hall current of anomalous parity.)

At a fundamental level, quantum anomalies are at the forefront of condensed matter physics and material science in the pursuit of topological phases of matters (topological quantum matters) first studied by Semenoff ${ }^{251}$ and Haldane $^{252}$ in lattices and narrow-gap semiconductors. The emergent anomalies are typically relativistic quantum phenomena and induce intriguing quantum-classical correspondence in terms of the underlying fundamental symmetries, which are then relevant to relativistic quantum chaos but not to traditional, nonrelativistic quantum chaos. When taking into account the intrinsic spin degree of freedom and the striking symmetry anomalies, the study of relativistic quantum chaos represents a new twist that does not exist in the study of traditional quantum chaos. Exciting new physics can be anticipated through the study of the manifestations of classical chaos in Dirac materials and topological quantum systems.

Taken together, the approaches to pertinent topics in relativistic quantum chaos and the various phenomena uncovered so far, as reviewed in this Tutorial, indicate that the field is not just some standard machinery of quantum chaos applied to relativistic quantum systems. From a fundamental point of view, due to uniquely relativistic quantum phenomena such as Klein tunneling, Zitterbewegung, and pair creations that find no counterparts in nonrelativistic quantum systems, strikingly new and counterintuitive phenomena can be uncovered in the pursuit of relativistic quantum chaos. From an applied perspective, the tremendous recent development of Dirac materials such as graphene and topological insulators provides a reason to investigate the effects of chaos on the physics of electronic, spintronic, and even valleytronic systems and to exploit chaos to improve device performance, further justifying the study of relativistic quantum chaos. There is potential that novel concepts and cutting-edge devices would arise from research on the classical-relativistic 
quantum correspondence. At the present, the interplay between classical chaos and fundamentally relativistic quantum entities and phenomena such as the intrinsic spin degree of freedom, valley degree of freedom, symmetry anomalies, and Klein tunneling, is largely unknown, demanding attention from the physics community to the emergent field of relativistic quantum chaos.

The emergent field of relativistic quantum chaos is not to be confused with the previous studies of classical nonlinear and chaotic behavior in the context of special or general relativity. ${ }^{155-159}$ Even with a focus on relativistic systems, these studies did not deal with the relativistic quantum manifestations of classical chaos. In particular, Ref. 155 addresses the chaotic geodesic scattering/motion in radiative plane wave spacetime characterized by a particular metric form through the deduction of an equivalent classical Hénon-Heiles Hamiltonian. In Ref. 156, the author studied the imaginary mass tachyon physics in open hyperbolic space with constant negative curvature, adopting the field theory in analogy to classical electrodynamics for photons. In this system, classical chaos arises due to the open, multiconnected, and hyperbolic cosmic space, and the quantum effect is treated by including the induced tachyon potential into the Schrödinger equation as a perturbation to the Coulomb potential in the local Euclidean limit. In Ref. 157, ideas from nonlinear dynamics were exploited to ascertain general relativistic dynamics, providing a new class of classical nonlinear physical systems with nonlinearity arising from complicated gravitational effects. Reference 158 treats a classical three-body problem, where the classical motions of a charged particle in a static electric field generated by two fixed, equal charges in space were studied in the context of special relativity. For this system, the emerging nonintegrable dynamics are due to the intrinsic nonlinear form of the relativistic description of the kinematics. In Ref. 159, the authors investigated classical chaotic scattering in general relativistic systems in the presence of a Yang-Mills field.

We emphasize that this Tutorial on relativistic quantum chaos deals with the relativistic quantum manifestations of classical chaos, which was stimulated by the vast research on graphene and the subsequent development of a large variety of Dirac materials including topological insulators. As reviewed in this Tutorial, the manifestations of classical chaos in condensed matter systems governed by relativistic quantum mechanics can be exploited for potential applications, e.g., modulating quantum transport in graphene and topological insulator devices through the pseudospin degree of freedom. Although relativistic quantum chaos is quite relevant to graphene, topological insulators, or any other $2 \mathrm{D}$ Dirac materials, this Tutorial is not just another review on graphene or topological insulators - it is on a new and emergent field in physics that treats the fundamental interplay between chaos and relativistic quantum mechanics.

\section{ACKNOWLEDGMENTS}

We would like to acknowledge support from the Vannevar Bush Faculty Fellowship program sponsored by the Basic Research Office of the Assistant Secretary of Defense for Research and Engineering and funded by the Office of Naval Research through Grant No. N00014-16-12828. L.H. was supported by the NSF of China under Grant No. 11422541.

\section{APPENDIX: DIRAC EQUATION IN A CLOSED DOMAIN AND BOUNDARY CONDITIONS}

Consider a massless spin- $1 / 2$ particle in a finite domain $D$ in the plane $\boldsymbol{r}=(x, y)$, as shown in the upper left corner of Fig. 10. Utilizing an infinite-mass term outside the domain to model the confinement of the particle motion within $D$, one obtains the following Dirac Hamiltonian in the position representation

$$
\hat{H}=-i \hbar v \hat{\sigma} \cdot \nabla+V(\boldsymbol{r}) \hat{\sigma}_{z},
$$

where $\hat{\sigma}=\left(\hat{\sigma}_{x}, \hat{\sigma}_{y}\right)$ and $\hat{\sigma}_{z}$ are Pauli matrices, and $v$ is the Fermi velocity. The Hamiltonian $\hat{H}$ acts on the twocomponent spinor wave-function $\psi(\boldsymbol{r})=\left[\psi_{1}, \psi_{2}\right]^{\mathrm{T}}$ and it has eigenvalue $E$, i.e.,

$$
\left[-i \hbar v \hat{\sigma} \cdot \nabla+V(\boldsymbol{r}) \hat{\sigma}_{z}\right] \psi(\boldsymbol{r})=E \psi(\boldsymbol{r}) .
$$

Some basic properties of Eq. (A2) are the following. First, the confinement condition of imposing an infinite mass outside $D$ naturally takes into account the Klein paradox for relativistic quantum particles. Second, the reduced spatial dimension and confinement break the time-reversal symmetry of $\hat{H}$

$$
[\hat{T}, \hat{H}] \neq 0
$$

where $\hat{T}=i \sigma_{y} \hat{K}$, and $\hat{K}$ denotes the complex conjugate. Third, for $V=0$ in Eq. (A2), there exist plane-wave solutions with the positive energy component given by

$$
\psi_{\boldsymbol{k}}(\boldsymbol{r})=\frac{1}{\sqrt{2}}\left(\begin{array}{c}
\exp \left(-i \frac{\theta}{2}\right) \\
\exp \left(i \frac{\theta}{2}\right)
\end{array}\right) \exp (i \boldsymbol{k} \cdot \boldsymbol{r})
$$

where $\boldsymbol{k}$ is a wave-vector that makes an angle $\theta$ with the $x$ axis.

To obtain solutions of Eq. (A2), a proper treatment of the boundary condition is necessary. As illustrated in the upper left corner of Fig. 10, we let the outward unit normal at $s$ be $\boldsymbol{n}(s)=[\cos (\theta), \sin (\theta)]$. Making use of the hermiticity of $\hat{H}$ and defining $\boldsymbol{j}=v_{F} \psi^{\dagger} \hat{\sigma} \psi$ as the local relativistic current, one gets the vanishing current condition: $\boldsymbol{j} \cdot \boldsymbol{n}=0$ for any point $s$. Requiring the outward current to be zero cannot fix the boundary condition uniquely but it entails $\operatorname{Re}\left(\exp (i \theta) \psi_{1} / \psi_{2}\right)=0$ for any point $s$. Using the boundary potential as in Ref. 96, one obtains the following complete boundary condition:

$$
\frac{\psi_{2}}{\psi_{1}}=i \exp [i \theta(s)] .
$$


${ }^{1}$ H.-J. Stöckmann, Quantum Chaos: An Introduction (Cambridge University Press, New York, 1999).

${ }^{2}$ F. Haake, Quantum Signatures of Chaos, Springer Series in Synergetics, 3rd ed. (Springer-Verlag, Berlin, 2010).

${ }^{3}$ O. Bohigas, M. J. Giannoni, and C. Schmit, "Characterization of chaotic quantum spectra and universality of level fluctuation laws," Phys. Rev. Lett. 52, 1-4 (1984).

${ }^{4}$ M. V. Berry, "Semiclassical theory of spectral rigidity," Proc. R. Soc. London, Ser. A 400, 229-251 (1985).

${ }^{5}$ M. Feingold, S. Fishman, D. R. Grempel, and R. E. Prange, "Statistics of quasi-energy separations in chaotic systems," Phys. Rev. B 31, 6852-6855 (1985).

${ }^{6}$ G. Casati, B. V. Chirikov, and I. Guarneri, "Energy-level statistics of integrable quantum systems," Phys. Rev. Lett. 54, 1350-1353 (1985).

${ }^{7}$ M. V. Berry and M. Robnik, "Statistics of energy-levels without timereversal symmetry-Aharonov-Bohm chaotic billiards," J. Phys. A Math. Gen. 19, 649-668 (1986).

${ }^{8} \mathrm{M}$. Feingold and S. Fishman, "Statistics of quasienergies in chaotic and random systems," Physica D 25, 181-195 (1987).

${ }^{9}$ B. V. Chirikov and D. L. Shepelyansky, "Shnirelman peak in level spacing statistics," Phys. Rev. Lett. 74, 518-521 (1995).

${ }^{10} \mathrm{O}$. Agam and S. Fishman, "Semiclassical analysis of energy level correlations for a disordered mesoscopic system," Phys. Rev. Lett. 76, 726-729 (1996).

${ }^{11}$ T. Kottos and U. Smilansky, "Quantum chaos on graphs," Phys. Rev. Lett. 79, 4794-4797 (1997).

${ }^{12}$ T. Kottos and U. Smilansky, "Periodic orbit theory and spectral statistics for quantum graphs,” Ann. Phys. 274, 76-124 (1999).

${ }^{13} \mathrm{~T}$. Dittrich et al., "Spectral correlations in systems undergoing a transition from periodicity to disorder," Phys. Rev. E 59, 6541-6551 (1999).

${ }^{14}$ B. Gutkin and U. Smilansky, "Can one hear the shape of a graph?," J. Phys. A 34, 6061-6068 (2001).

${ }^{15}$ S. Rahav and S. Fishman, "Spectral statistics of rectangular billiards with localizedperturbations," Nonlinearity 15, 1541-1594 (2002).

${ }^{16}$ G. Blum, S. Gnutzmann, and U. Smilansky, "Nodal domains statistics: A criterion for quantum chaos,” Phys. Rev. Lett. 88, 114101 (2002).

${ }^{17}$ S. Gnutzmann and U. Smilansky, "Quantum graphs: Applications to quantum chaos and universal spectral statistics," Adv. Phys. 55, 527-625 (2006).

${ }^{18}$ S. Gnutzmann, P. D. Karageorge, and U. Smilansky, "Can one count the shape of a drum?," Phys. Rev. Lett. 97, 090201 (2006).

${ }^{19}$ U. Smilansky, "Quantum chaos on discrete graphs," J. Phys. A 40, F621-F630 (2007).

${ }^{20} \mathrm{~S}$. W. Mcdonald and A. N. Kaufman, "Spectrum and eigenfunctions for a Hamiltonian with stochastic trajectories," Phys. Rev. Lett. 42, 1189-1191 (1979).

${ }^{21}$ E. J. Heller, "Bound-state eigenfunctions of classically chaotic Hamiltonian systems-Scars of periodic orbits," Phys. Rev. Lett. 53, 1515-1518 (1984)

${ }^{22}$ B. V. Chirikov, "An example of chaotic eigenstates in a complex atom," Phys. Lett. A 108, 68-70 (1985).

${ }^{23}$ S. W. Mcdonald and A. N. Kaufman, "Wave chaos in the stadiumStatistical properties of short-wave solutions of the Helmholtz equation," Phys. Rev. A 37, 3067-3086 (1988).

${ }^{24}$ E. B. Bogomolny, "Smoothed wave-functions of chaotic quantum systems," Physica D 31, 169-189 (1988).

${ }^{25}$ R. L. Waterland, J. M. Yuan, C. C. Martens, R. E. Gillilan, and W. P. Reinhardt, "Classical-quantum correspondence in the presence of global chaos," Phys. Rev. Lett. 61, 2733-2736 (1988).

${ }^{26}$ M. V. Berry, "Quantum scars of classical closed orbits in phase-space," Proc. R. Soc. London, Ser. A 423, 219-231 (1989).

${ }^{27}$ B. Eckhardt, G. Hose, and E. Pollak, "Quantum-mechanics of a classically chaotic system-Observations on scars, periodic-orbits, and vibrational adiabaticity," Phys. Rev. A 39, 3776-3793 (1989).

${ }^{28}$ R. V. Jensen, M. M. Sanders, M. Saraceno, and B. Sundaram, "Inhibition of quantum transport due to scars of unstable periodic-orbits," Phys. Rev. Lett. 63, 2771-2775 (1989).

${ }^{29}$ H. J. Stockmann and J. Stein, "Quantum chaos in billiards studied by microwave-absorption," Phys. Rev. Lett. 64, 2215-2218 (1990).

${ }^{30}$ B. Eckhardt, J. M. G. Llorente, and E. Pollak, "Phase-space analysis of chaotic spectra in a conservative Hamiltonian system," Chem. Phys. Lett. 174, 325-332 (1990).
${ }^{31}$ R. Blümel, I. H. Davidson, W. P. Reinhardt, H. Lin, and M. Sharnoff, "Quasi-linear ridge structures in water-surface waves," Phys. Rev. A 45, 2641-2644 (1992).

${ }^{32}$ M. Kus, J. Zakrzewski, and K. Zyczkowski, "Quantum scars on a sphere," Phys. Rev. A 43, 4244-4248 (1991).

${ }^{33}$ R. V. Jensen, "Quantum chaos," Nature 355, 311-318 (1992).

${ }^{34} \mathrm{R}$. V. Jensen, "Quantum mechanics-Bringing order out of chaos," Nature 355, 591-592 (1992).

${ }^{35} \mathrm{O}$. Agam and S. Fishman, "Quantum eigenfunctions in terms of periodic orbits of chaotic systems," J. Phys. A 26, 2113-2137 (1993).

${ }^{36}$ O. Agam and S. Fishman, "Semiclassical criterion for scars in wave functions of chaotic systems," Phys. Rev. Lett. 73, 806-809 (1994).

${ }^{37}$ B. Eckhardt et al., "Approach to ergodicity in quantum wave functions," Phys. Rev. E 52, 5893-5903 (1995).

${ }^{38}$ T. S. Monteiro, D. Delande, and J. P. Connerade, "Have quantum scars been observed?," Nature 387, 863-864 (1997).

${ }^{39}$ C. P. Malta, M. A. M. Deaguiar, and A. M. O. Dealmeida, "Quantum signature of a period-doubling bifurcation and scars of periodic orbits," Phys. Rev. A 47, 1625-1632 (1993).

${ }^{40}$ G. G. Depolavieja, F. Borondo, and R. M. Benito, "Scars in groups of eigenstates in a classically chaotic system," Phys. Rev. Lett. 73, 1613-1616 (1994).

${ }^{41}$ T. M. Fromhold et al., "Manifestations of classical chaos in the energylevel spectrum of a quantum-well," Phys. Rev. Lett. 75, 1142-1145 (1995).

${ }^{42}$ P. Bellomo and T. Uzer, "Quantum scars and classical ghosts," Phys. Rev. A 51, 1669-1672 (1995).

${ }^{43} \mathrm{O}$. Agam, "Quantum scars of classical orbits in small interacting electronic systems," Phys. Rev. B 54, 2607-2628 (1996).

${ }^{44}$ S. Fishman, B. Georgeot, and R. E. Prange, "Fredholm method for scars," J. Phys. A 29, 919-937 (1996).

${ }^{45}$ R. Akis, D. K. Ferry, and J. P. Bird, "Wave function scarring effects in open stadium shaped quantum dots," Phys. Rev. Lett. 79, 123-126 (1997).

${ }^{46}$ F. P. Simonotti, E. Vergini, and M. Saraceno, "Quantitative study of scars in the boundary section of the stadium billiard," Phys. Rev. E 56, 3859-3867 (1997).

${ }^{47}$ L. Kaplan and E. J. Heller, "Linear and nonlinear theory of eigenfunction scars," Ann. Phys. 264, 171-206 (1998).

${ }^{48}$ E. E. Narimanov and A. D. Stone, "Origin of strong scarring of wave functions in quantum wells in a tilted magnetic field," Phys. Rev. Lett. 80, 49-52 (1998).

${ }^{49}$ L. Kaplan, "Scars in quantum chaotic wavefunctions," Nonlinearity $\mathbf{1 2}$, R1-R40 (1999).

${ }^{50} \mathrm{O}$. M. Auslaender and S. Fishman, "Exact eigenfunctions of a chaotic system," Physica D 128, 180-223 (1999).

${ }^{51}$ J. P. Keating and S. D. Prado, "Orbit bifurcations and the scarring of wave functions," Proc. R. Soc. A 457, 1855-1872 (2001).

${ }^{52} \mathrm{H}$. Schanz and T. Kottos, "Scars on quantum networks ignore the Lyapunov exponent," Phys. Rev. Lett. 90, 234101 (2003).

${ }^{53}$ R. Blümel and U. Smilansky, "Classical irregular scattering and its quantum-mechanical implications," Phys. Rev. Lett. 60, 477-480 (1988).

${ }^{54}$ R. Blümel and U. Smilansky, "A simple-model for chaotic scattering 2. quantum-mechanical theory," Physica D 36, 111-136 (1989).

${ }^{55}$ R. A. Jalabert, H. U. Baranger, and A. D. Stone, "Conductance fluctuations in the ballistic regime-A probe of quantum chaos," Phys. Rev. Lett. 65, 2442-2445 (1990).

${ }^{56}$ C. M. Marcus, A. J. Rimberg, R. M. Westervelt, P. F. Hopkins, and A. C. Gossard, "Conductance fluctuations and chaotic scattering in ballistic microstructures," Phys. Rev. Lett. 69, 506-509 (1992).

${ }^{57}$ Y.-C. Lai, R. Blümel, E. Ott, and C. Grebogi, "Quantum manifestations of chaotic scattering," Phys. Rev. Lett. 68, 3491-3494 (1992).

${ }^{58}$ R. Ketzmerick, "Fractal conductance fluctuations in generic chaotic cavities," Phys. Rev. B 54, 10841-10844 (1996).

${ }^{59}$ A. S. Sachrajda et al., "Fractal conductance fluctuations in a soft-wall stadium and a Sinai billiard," Phys. Rev. Lett. 80, 1948-1951 (1998).

${ }^{60}$ T. Kottos, U. Smilansky, J. Fortuny, and G. Nesti, "Chaotic scattering of microwaves," Radio Sci. 34, 747-758, https://doi.org/10.1029/ 1999RS900037 (1999).

${ }^{61}$ T. Kottos and U. Smilansky, "Chaotic scattering on graphs," Phys. Rev. Lett. 85, 968-971 (2000).

${ }^{62}$ B. Huckestein, R. Ketzmerick, and C. H. Lewenkopf, "Quantum transport through ballistic cavities: Soft vs hard quantum chaos," Phys. Rev. Lett. 84, 5504-5507 (2000). 
${ }^{63}$ G. Casati, I. Guarneri, and G. Maspero, "Fractal survival probability fluctuations," Phys. Rev. Lett. 84, 63-66 (2000).

${ }^{64}$ A. P. S. de Moura, Y.-C. Lai, R. Akis, J. P. Bird, and D. K. Ferry, "Tunneling and nonhyperbolicity in quantum dots," Phys. Rev. Lett. 88, 236804 (2002).

${ }^{65} \mathrm{R}$. Crook et al., "Imaging fractal conductance fluctuations and scarred wave functions in a quantum billiard," Phys. Rev. Lett. 91, 246803 (2003).

${ }^{66}$ T. Kottos and U. Smilansky, "Quantum graphs: A simple model for chaotic scattering," J. Phys. A 36, 3501-3524 (2003).

${ }^{67}$ R. Band, A. Sawicki, and U. Smilansky, "Scattering from isospectral quantum graphs," J. Phys. A 43, 415201 (2010).

${ }^{68}$ Y. Krivolapov, S. Fishman, E. Ott, and T. M. Antonsen, "Quantum chaos of a mixed open system of kicked cold atoms," Phys. Rev. E 83, 016204 (2011).

${ }^{69}$ S. Fishman, D. R. Grempel, and R. E. Prange, "Chaos, quantum recurrences, and Anderson localization," Phys. Rev. Lett. 49, 509-512 (1982).

${ }^{70}$ G. Casati, B. V. Chirikov, and D. L. Shepelyansky, "Quantum limitations for chaotic excitation of the hydrogen atom in a monochromatic field," Phys. Rev. Lett. 53, 2525-2528 (1984).

${ }^{71}$ G. Casati, B. V. Chirikov, I. Guarneri, and D. L. Shepelyansky, "Dynamical stability of quantum "chaotic" motion in a hydrogen atom," Phys. Rev. Lett. 56, 2437-2440 (1986).

${ }^{72}$ G. Casati, B. V. Chirikov, D. L. Shepelyansky, and I. Guarneri, "New photoelectric ionization peak in the hydrogen atom," Phys. Rev. Lett. 57, 823-826 (1986).

${ }^{73}$ G. Casati, B. V. Chirikov, D. L. Shepelyansky, and I. Guarneri, "Relevance of classical chaos in quantum mechanics: The hydrogen atom in a monochromatic field," Phys. Rep. 154, 77-123 (1987).

${ }^{74}$ G. Casati, B. V. Chirikov, I. Guarneri, and D. L. Shepelyansky, "Localization of diffusive excitation in the two-dimensional hydrogen atom in a monochromatic field," Phys. Rev. Lett. 59, 2927-2930 (1987).

${ }^{75}$ B. V. Chirikov, F. M. Izrailev, and D. L. Shepelyansky, "Quantum chaos: Localization vs. ergodicity," Physica D 33, 77-88 (1988).

${ }^{76}$ J. E. Bayfield, G. Casati, I. Guarneri, and D. W. Sokol, "Localization of classically chaotic diffusion for hydrogen atoms in microwave fields," Phys. Rev. Lett. 63, 364-367 (1989).

${ }^{77}$ B. V. Chirikov, "A theory of quantum diffusion localization," Chaos $\mathbf{1}$, 95-100 (1991)

${ }^{78}$ B. V. Chirikov, Chaos and Quantum Mechanics, Les Houches Lecture Series Vol. 52, edited by M.-J. Giannoni, A. Voros, and J. Zinn-Justin (Elsevier Sci. Publ., Amsterdam, 1991), pp. 443-545.

${ }^{79}$ G. Casati, B. V. Chirikov, I. Guarneri, and F. M. Izrailev, "Band-randommatrix model for quantum localization in conservative systems," Phys. Rev. E 48, R1613-R1616 (1993).

${ }^{80}$ G. Casati and B. V. Chirikov, "Quantum chaos: Unexpected complexity," Physica D 86, 220-237 (1995)

${ }^{81}$ G. Casati, B. V. Chirikov, I. Guarneri, and F. M. Izrailev, "Quantum ergodicity and localization in conservative systems: The Wigner band random matrix model," Phys. Lett. A 223, 430-435 (1996).

${ }^{82}$ G. Casati, B. V. Chirikov, and O. V. Zhirov, "Existence of a long time scale in quantum chaos," Phys. Rev. E 55, 7757-7758 (1997).

${ }^{83}$ A. Iomin, S. Fishman, and G. M. Zaslavsky, "Quantum localization for a kicked rotor with accelerator mode islands," Phys. Rev. E 65, 036215 (2002).

${ }^{84}$ A. Iomin, S. Fishman, and G. M. Zaslavsky, "Semiclassical quantization of separatrix maps," Phys. Rev. E 67, 046210 (2003).

${ }^{85} \mathrm{~B}$. V. Chirikov, "A universal instability of many-dimensional oscillator systems," Phys. Rep. 52, 263-379 (1979).

${ }^{86}$ R. V. Jensen, S. M. Susskind, and M. M. Sanders, "Chaotic ionization of highly excited hydrogen atoms: Comparison of classical and quantum theory with experiment," Phys. Rep. 201, 1-56 (1991).

${ }^{87}$ P. M. Koch and K. H. A. van Leeuwen, "The importance of resonances in microwave "ionization" of excited hydrogen atoms," Phys. Rep. 256, 289-403 (1995).

${ }^{88}$ P. J. J. Luukko et al., "Strong quantum scarring by local impurities," Sci. Rep. 6, 37656 (2016).

${ }^{89}$ S. Fishman, R. E. Prange, and M. Griniasty, "Scaling theory for the localization length of the kicked rotor," Phys. Rev. A 39, 1628-1633 (1989).

${ }^{90}$ R. E. Prange and S. Fishman, "Experimental realizations of kicked quantum chaotic systems," Phys. Rev. Lett. 63, 704-707 (1989).

${ }^{91}$ R. Blümel and U. Smilansky, "Ionization of excited hydrogen atoms by microwave fields: A test case for quantum chaos," Phys. Scr. 40, 386-393 (1989).
${ }^{92}$ M. Robnik and M. V. Berry, "False time-reversal violation and energy level statistics: The role of anti-unitary symmetry," J. Phys. A 19, 669 (1986).

${ }^{93}$ D. R. Grempel, S. Fishman, and R. E. Prange, "Localization in an incommensurate potential: An exactly solvable model," Phys. Rev. Lett. 49, 833-836 (1982).

${ }^{94}$ R. E. Prange, D. R. Grempel, and S. Fishman, "Solvable model of quantum motion in an incommensurate potential," Phys. Rev. B 29, 6500-6512 (1984)

${ }^{95}$ M. V. Berry, "Incommensurability in an exactly-soluble quantal and classical model for a kicked rotator," Physica D 10, 369-378 (1984).

${ }^{96} \mathrm{M}$. V. Berry and R. J. Mondragon, "Neutrino billiards-Time-reversal symmetry-breaking without magnetic-fields," Proc. R. Soc. London, Ser. A 412, 53-74 (1987).

${ }^{97}$ M. Antoine, A. Comtet, and M. Knecht, "Heat kernel expansion for fermionic billiards in an external magnetic field," J. Phys. A 23, L35 (1990).

${ }^{98}$ R. Tomaschitz, "Relativistic quantum chaos in Robertson-Walker cosmologies," J. Math. Phys. 32, 2571-2579 (1991).

${ }^{99}$ E. Calzetta and J. J. Gonzalez, "Chaos and semiclassical limit in quantum cosmology," Phys. Rev. D 51, 6821-6828 (1995).

${ }^{100}$ N. J. Cornish and E. P. S. Shellard, "Chaos in quantum cosmology," Phys. Rev. Lett. 81, 3571-3574 (1998).

${ }^{101}$ M. Koehn, "Relativistic wavepackets in classically chaotic quantum cosmological billiards," Phys. Rev. D 85, 063501 (2012).

${ }^{102}$ M. A. Halasz and J. J. M. Verbaarschot, "Universal fluctuations in spectra of the lattice Dirac operator," Phys. Rev. Lett. 74, 3920-3923 (1995).

${ }^{103}$ B. A. Berg, H. Markum, and R. Pullirsch, "Quantum chaos in compact lattice QED,” Phys. Rev. D 59, 097504 (1999).

${ }^{104} \mathrm{~J}$. Bolte and J. Harrison, "Spectral statistics for the dirac operator on graphs," J. Phys. A 36, 2747 (2003).

${ }^{105}$ S. C. Phatak, S. Pal, and D. Biswas, "Semiclassical features in the quantum description of a Dirac particle in a cavity," Phys. Rev. E 52, 1333-1344 (1995).

${ }^{106}$ A. H. C. Neto and K. Novoselov, "Two-dimensional crystals: Beyond graphene," Mater. Express 1, 10-17 (2011).

${ }^{107}$ P. Ajayan, P. Kim, and K. Banerjee, "Two-dimensional van der Waals materials," Phys. Today 69(9), 38-44 (2016).

${ }^{108}$ K. S. Novoselov et al., "Electric field effect in atomically thin carbon films," Science 306, 666-669 (2004).

${ }^{109} \mathrm{C}$. Berger et al., "Ultrathin epitaxial graphite: $2 \mathrm{D}$ electron gas properties and a route toward graphene-based nanoelectronics," J. Phys. Chem. B 108, 19912-19916 (2004).

${ }^{110} \mathrm{~K}$. S. Novoselov et al., "Two-dimensional gas of massless Dirac fermions in graphene," Nature 438, 197-200 (2005).

${ }^{111}$ Y. B. Zhang, Y. W. Tan, H. L. Stormer, and P. Kim, "Experimental observation of the quantum Hall effect and Berry's phase in graphene," Nature 438, 201-204 (2005).

${ }^{112}$ A. H. C. Neto, F. Guinea, N. M. R. Peres, K. S. Novoselov, and A. K. Geim, "The electronic properties of graphene," Rev. Mod. Phys. 81, 109-162 (2009).

${ }^{113}$ N. M. R. Peres, "Colloquium: The transport properties of graphene: An introduction,” Rev. Mod. Phys. 82, 2673-2700 (2010).

${ }^{114}$ S. D. Sarma, S. Adam, E. H. Hwang, and E. Rossi, "Electronic transport in two-dimensional graphene," Rev. Mod. Phys. 83, 407-470 (2011).

${ }^{115}$ M. Z. Hasan and C. L. Kane, "Colloquium: Topological insulators," Rev. Mod. Phys. 82, 3045-3067 (2010).

${ }^{116}$ B. Radisavljevic, A. Radenovic, J. Brivio, V. Giacometti, and A. Kis, "Single-layer $\mathrm{MoS}_{2}$ transistors," Nat. Nanotechnol. 6, 147-150 (2011).

${ }^{117}$ Q. H. Wang, K. Kalantar-Zadeh, A. Kis, J. N. Coleman, and M. S. Strano, "Electronics and optoelectronics of two-dimensional transition metal dichalcogenides," Nat. Nanotechnol. 7, 699-712 (2012).

${ }^{118} \mathrm{D}$. Sheberla et al., "High electrical conductivity in $\mathrm{ni}_{3}(2,3,6,7,10,11$-hexaiminotriphenylene $)_{2}-\mathrm{A}$ semiconducting metal-organic graphene analogue," J. Am. Chem. Soc. 136, 8859-8862 (2014).

${ }^{119}$ Z. K. Liu et al., "Discovery of a three-dimensional topological Dirac semimetal $\mathrm{Na}_{3} \mathrm{Bi}$," Science 343, 864-867 (2014).

${ }^{120} \mathrm{Z}$. K. Liu et al., "A stable three-dimensional topological Dirac semimetal $\mathrm{Cd}_{3} \mathrm{As}_{2}$," Nat. Mater. 13, 677-681 (2014).

${ }^{121}$ L. Huang, Y.-C. Lai, D. K. Ferry, S. M. Goodnick, and R. Akis, "Relativistic quantum scars," Phys. Rev. Lett. 103, 054101 (2009).

${ }^{122}$ J. H. Bardarson, M. Titov, and P. W. Brouwer, "Electrostatic confinement of electrons in an integrable graphene quantum dot," Phys. Rev. Lett. 102, 226803 (2009). 
${ }^{123}$ L. Huang, Y.-C. Lai, and C. Grebogi, "Relativistic quantum levelspacing statistics in chaotic graphene billiards," Phys. Rev. E 81, 055203 (2010).

${ }^{124}$ L. L. Jiang, L. Huang, R. Yang, and Y.-C. Lai, "Control of transmission in disordered graphene nanojunctions through stochastic resonance," Appl. Phys. Lett. 96, 262114 (2010).

${ }^{125}$ M. Wimmer, A. R. Akhmerov, and F. Guinea, "Robustness of edge states in graphene quantum dots," Phys. Rev. B 82, 045409 (2010).

${ }^{126}$ K. Wakabayashi, S. Okada, R. Tomita, S. Fujimoto, and Y. Natsume, "Edge states and flat bands of graphene nanoribbons with edge modification,” J. Phys. Soc. Jpn. 79, 034706 (2010).

${ }^{127}$ L. Huang, Y.-C. Lai, and C. Grebogi, "Characteristics of level-spacing statistics in chaotic graphene billiards," Chaos 21, 013102 (2011).

${ }^{128}$ R. Yang, L. Huang, Y.-C. Lai, and C. Grebogi, "Quantum chaotic scattering in graphene systems," Europhys. Lett. 94, 40004 (2011).

${ }^{129}$ M. Schneider and P. W. Brouwer, "Resonant scattering in graphene with a gate-defined chaotic quantum dot," Phys. Rev. B 84, 115440 (2011).

${ }^{130} \mathrm{~A}$. Rycerz, "Random matrices and quantum chaos in weakly disordered graphene nanoflakes," Phys. Rev. B 85, 245424 (2012).

${ }^{131}$ R. Yang, L. Huang, Y.-C. Lai, and L. M. Pecora, "Modulating quantum transport by transient chaos," Appl. Phys. Lett. 100, 093105 (2012).

${ }^{132}$ L. Ying, L. Huang, Y.-C. Lai, and C. Grebogi, "Conductance fluctuations in graphene systems: The relevance of classical dynamics," Phys. Rev. B 85, 245448 (2012).

${ }^{133}$ X. Ni, L. Huang, Y.-C. Lai, and L. M. Pecora, "Effect of chaos on relativistic quantum tunneling," Europhys. Lett. 98, 50007 (2012).

${ }^{134} \mathrm{X}$. Ni, L. Huang, Y.-C. Lai, and C. Grebogi, "Scarring of Dirac fermions in chaotic billiards," Phys. Rev. E 86, 016702 (2012).

${ }^{135}$ J. Heinl, M. Schneider, and P. W. Brouwer, "Interplay of AharonovBohm and Berry phases in gate-defined graphene quantum dots," Phys. Rev. B 87, 245426 (2013).

${ }^{136}$ H. Y. Xu, L. Huang, Y.-C. Lai, and C. Grebogi, "Chiral scars in chaotic Dirac fermion systems," Phys. Rev. Lett. 110, 064102 (2013).

${ }^{137}$ L. Huang, R. Yang, Y.-C. Lai, and D. K. Ferry, "Lead-position dependent regular oscillations and random fluctuations of conductance in graphene quantum dots," J. Phys. Condens. Matter 25, 085502 (2013).

${ }^{138}$ L. Ying, L. Huang, Y.-C. Lai, and Y. Zhang, "Effect of geometrical rotation on conductance fluctuations in graphene quantum dots," J. Phys. Condens. Matter 25, 105802 (2013).

${ }^{139}$ R. Yang, L. Huang, Y.-C. Lai, C. Grebogi, and L. M. Pecora, "Harnessing quantum transport by transient chaos," Chaos 23, 013125 (2013).

${ }^{140}$ G. L. Wang, L. Ying, Y.-C. Lai, and C. Grebogi, "Quantum chaotic scattering in graphene systems in the absence of invariant classical dynamics," Phys. Rev. E 87, 052908 (2013).

${ }^{141}$ X. Ni, L. Huang, L. Ying, and Y.-C. Lai, "Relativistic quantum tunneling of a Dirac fermion in nonhyperbolic chaotic systems," Phys. Rev. B 87, 224304 (2013)

${ }^{142}$ M. S. M. Barros, A. J. N. Júnior, A. F. Macedo-Junior, J. G. G. S. Ramos, and A. L. R. Barbosa, "Open chaotic Dirac billiards: Weak (anti)localization, conductance fluctuations, and decoherence," Phys. Rev. B 88, 245133 (2013)

${ }^{143}$ L. Ying, G. Wang, L. Huang, and Y.-C. Lai, "Quantum chaotic tunneling in graphene systems with electron-electron interactions," Phys. Rev. B 90, 224301 (2014)

${ }^{144} \mathrm{M}$. Schneider and P. W. Brouwer, "Density of states as a probe of electrostatic confinement in graphene," Phys. Rev. B 89, 205437 (2014).

${ }^{145}$ J. G. G. S. Ramos, I. M. L. da Silva, and A. L. R. Barbosa, "Anomalous entanglement in chaotic Dirac billiards," Phys. Rev. B 90, 245107 (2014).

${ }^{146}$ H.-Y. Xu, L. Huang, Y.-C. Lai, and C. Grebogi, "Superpersistent currents and whispering gallery modes in relativistic quantum chaotic systems," Sci. Rep. 5, 8963 (2015)

${ }^{147}$ H.-Y. Xu and Y.-C. Lai, "Reverse Stark effect, anomalous optical transitions, and control of spin in topological insulator quantum dots," Phys. Rev. B 92, 195120 (2015).

${ }^{148}$ H. Xu, L. Huang, and Y.-C. Lai, "A robust relativistic quantum two-level system with edge-dependent currents and spin polarization," Europhys. Lett. 115, 20005 (2016).

${ }^{149}$ J. G. G. S. Ramos, M. S. Hussein, and A. L. R. Barbosa, "Fluctuation phenomena in chaotic Dirac quantum dots: Artificial atoms on graphene flakes,” Phys. Rev. B 93, 125136 (2016).

${ }^{150}$ T. C. Vasconcelos, J. G. G. S. Ramos, and A. L. R. Barbosa, "Universal spin Hall conductance fluctuations in chaotic Dirac quantum dots," Phys. Rev. B 93, 115120 (2016).
${ }^{151}$ L. Ying and Y.-C. Lai, "Enhancement of spin polarization by chaos in graphene quantum dot systems," Phys. Rev. B 93, 085408 (2016).

${ }^{152}$ I. Hagymási, P. Vancsó, A. Pálinkás, and Z. Osváth, "Interaction effects in a chaotic graphene quantum billiard," Phys. Rev. B 95, 075123 (2017).

${ }^{153}$ C.-Z. Wang, L. Huang, and K. Chang, "Scars in Dirac fermion systems: The influence of an Aharonov-Bohm flux," New J. Phys. 19, 013018 (2017).

${ }^{154}$ R. Yang, L. Huang, Y.-C. Lai, and C. Grebogi, "Abnormal electron paths induced by Klein tunneling in graphene quantum point contacts," Phys. Rev. B 84, 035426 (2011).

${ }^{155}$ J. C V. Podolský and K. Veselý, "Chaos in pp-wave spacetimes," Phys. Rev. D 58, 081501 (1998).

${ }^{156}$ R. Tomaschitz, "Tachyons, Lamb shifts and superluminal chaos," Euro. Phys. J. B 17, 523-536 (2000).

${ }^{157}$ J. D. Barrow, "General relativistic chaos and nonlinear dynamics," Gen. Relativ. Gravitation 14, 523-530 (1982).

${ }^{158}$ S. P. Drake, C. P. Dettmann, N. E. Frankel, and N. J. Cornish, "Chaos in special relativistic dynamics," Phys. Rev. E 53, 1351-1361 (1996).

${ }^{159}$ J. D. Barrow and J. Levin, "Chaos in the Einstein-Yang-Mills equations," Phys. Rev. Lett. 80, 656-659 (1998).

${ }^{160}$ D. Cabosart et al., "Recurrent quantum scars in a mesoscopic graphene ring," Nano Lett. 17, 1344-1349 (2017).

${ }^{161}$ M. C. Gutzwiller, "Periodic orbits and classical quantization conditions," J. Math. Phys. 12, 343-358 (1971).

${ }^{162} \mathrm{H}$. Zhang et al., "Topological insulators in $\mathrm{Bi}_{2} \mathrm{Se}_{3}, \mathrm{Bi}_{2} \mathrm{Te}_{3}$ and $\mathrm{Sb}_{2} \mathrm{Te}_{3}$ with a single Dirac cone on the surface," Nat. Phys. 5, 438-442 (2009).

${ }^{163}$ Y. L. Chen et al., "Massive Dirac fermion on the surface of a magnetically doped topological insulator," Science 329, 659-662 (2010).

${ }^{164} \mathrm{P}$. Wei et al., "Exchange-coupling-induced symmetry breaking in topological insulators," Phys. Rev. Lett. 110, 186807 (2013).

${ }^{165} \mathrm{M}$. Lang et al., "Proximity induced high-temperature magnetic order in topological insulator-Ferrimagnetic insulator heterostructure," Nano Lett. 14, 3459-3465 (2014).

${ }^{166}$ D. Gall, "Electron mean free path in elemental metals," J. Appl. Phys. 119, 085101 (2016).

${ }^{167}$ R. Landauer, "Spatial variation of currents and fields due to localized scatterers in metallic conduction," IBM J. Res. Dev. 1, 223-231 (1957).

${ }^{168}$ S. Datta, Electronic Transport in Mesoscopic Systems (Cambridge University Press, Cambridge, England, 1995).

${ }^{169}$ Y. V. Nazarov and Y. M. Blanter, Quantum Transport: Introduction to Nanoscience (Cambridge University Press, Cambridge, England, 2009).

${ }^{170}$ C. P. Umbach, S. Washburn, R. B. Laibowitz, and R. A. Webb, "Magnetoresistance of small, quasi-one-dimensional, normal-metal rings and lines," Phys. Rev. B 30, 4048-4051 (1984).

${ }^{171}$ P. A. Lee and A. D. Stone, "Universal conductance fluctuations in metals," Phys. Rev. Lett. 55, 1622-1625 (1985).

${ }^{172}$ R. A. Webb, S. Washburn, C. P. Umbach, and R. B. Laibowitz, "Observation of h/e Aharonov-Bohm oscillations in normal-metal rings," Phys. Rev. Lett. 54, 2696-2699 (1985).

${ }^{173}$ A. D. Stone, "Magnetoresistance fluctuations in mesoscopic wires and rings," Phys. Rev. Lett. 54, 2692-2695 (1985).

${ }^{174}$ B. L. Altshuler, "Fluctuations in the extrinsic conductivity of disordered conductors," JETP Lett. 41, 648 (1985); available at http://adsabs.harvard.edu/ abs/1985JETPL..41..648A.

${ }^{175} \mathrm{~S}$. V. Morozov et al., "Strong suppression of weak localization in graphene," Phys. Rev. Lett. 97, 016801 (2006).

${ }^{176} \mathrm{C}$. Berger et al., "Electronic confinement and coherence in patterned epitaxial graphene," Science 312, 1191-1196 (2006).

${ }^{177}$ H. B. Heersche, P. Jarillo-Herrero, J. B. Oostinga, L. M. K. Vandersypen, and A. F. Morpurgo, "Bipolar supercurrent in graphene," Nature 446, 56-59 (2007).

${ }^{178}$ A. Rycerz, J. Tworzydło, and C. Beenakker, “Anomalously large conductance fluctuations in weakly disordered graphene," Europhys. Lett. 79, 57003 (2007)

${ }^{179}$ D. W. Horsell, A. K. Savchenko, F. V. Tikhonenko, K. Kechedzhi, and I. V. Lerner, "Mesoscopic conductance fluctuations in graphene," Solid State Commun. 149, 1041-1045 (2009).

${ }^{180}$ D. W. Horsell, A. K. Savchenko, F. V. Tikhonenko, K. Kechedzhi, and I. V. Lerner, "Superconductivity-enhanced conductance fluctuations in fewlayer graphene," Nanotechnology 21, 274005 (2010); available at http:// iopscience.iop.org/article/10.1088/0957-4484/21/27/274005.

${ }^{181}$ R. Bao, L. Huang, Y.-C. Lai, and C. Grebogi, "Conductance fluctuations in chaotic bilayer graphene quantum dots," Phys. Rev. E 92, 012918 (2015). 
${ }^{182}$ U. Fano, "Effects of configuration interaction on intensities and phase shifts," Phys. Rev. 124, 1866-1878 (1961).

${ }^{183}$ A. E. Miroshnichenko, S. Flach, and Y. S. Kivshar, "Fano resonances in nanoscale structures," Rev. Mod. Phys. 82, 2257-2298 (2010).

${ }^{184}$ Y. Yoon et al., "Coupling quantum states through a continuum: A mesoscopic multistate Fano resonance," Phys. Rev. X 2, 021003 (2012); available at https://journals.aps.org/prx/abstract/10.1103/PhysRevX.2.021003.

${ }^{185} \mathrm{~J}$. Fransson et al., "Tuning the Fano resonance with an intruder continuum," Nano Lett. 14, 788-793 (2014).

${ }^{186}$ L. Huang, Y.-C. Lai, H.-G. Luo, and C. Grebogi, "Universal formalism of Fano resonance," AIP Adv. 5, 017137 (2015).

${ }^{187} \mathrm{P}$. Blanchard and R. Olkiewicz, "Decoherence-induced continuous pointer states," Phys. Rev. Lett. 90, 010403 (2003).

${ }^{188} \mathrm{~W}$. H. Zurek, "Decoherence, einselection, and the quantum origins of the classical," Rev. Mod. Phys. 75, 715-775 (2003).

${ }^{189}$ D. K. Ferry, R. Akis, and J. P. Bird, "Einselection in action: Decoherence and pointer states in open quantum dots," Phys. Rev. Lett. 93, 026803 (2004).

${ }^{190}$ R. Brunner, R. Akis, D. K. Ferry, F. Kuchar, and R. Meisels, "Couplinginduced bipartite pointer states in arrays of electron billiards: Quantum Darwinism in action?," Phys. Rev. Lett. 101, 024102 (2008).

${ }^{191}$ D. K. Ferry, L. Huang, R. Yang, Y.-C. Lai, and R. Akis, "Open quantum dots in graphene: Scaling relativistic pointer states," J. Phys. Conf. Ser. 220, 012015 (2010).

${ }^{192}$ M. Zwolak and W. H. Zurek, "Complementarity of quantum discord and classically accessible information," Sci. Rep. 3, 1729 (2013).

${ }^{193}$ H.-Y. Xu, G.-L. Wang, L. Huang, and Y.-C. Lai, "Chaos in Dirac electron optics: Emergence of a relativistic quantum chimera," Phys. Rev. Lett. 120, 124101 (2018).

${ }^{194}$ L. M. Pecora et al., "Chaos regularization of quantum tunneling rates," Phys. Rev. E 83, 065201 (2011).

${ }^{195}$ M. J. Lee, T. M. Antonsen, E. Ott, and L. M. Pecora, "Theory of chaos regularization of tunneling in chaotic quantum dots," Phys. Rev. E 86, 056212 (2012)

${ }^{196}$ M. I. Katsnelson, K. S. Novoselov, and A. K. Geim, "Chiral tunnelling and the Klein paradox in graphene," Nat. Phys. 2, 620-625 (2006).

${ }^{197}$ K. Wakabayashi and M. Sigrist, "Enhanced conductance fluctuation due to the zero-conductance Fano resonance in the quantum point contact on graphene,” J. Phys. Soc. Jpn. 77, 113708 (2008).

${ }^{198}$ M. Büttiker, Y. Imry, and R. Landauer, "Josephson behavior in small normal one-dimensional rings," Phys. Lett. A 96, 365-367 (1983).

${ }^{199}$ Y. Aharonov and D. Bohm, "Significance of electromagnetic potentials in the quantum theory," Phys. Rev. 115, 485 (1959).

${ }^{200}$ H.-F. Cheung, E. K. Riedel, and Y. Gefen, "Persistent currents in mesoscopic rings and cylinders,” Phys. Rev. Lett. 62, 587-590 (1989).

${ }^{201}$ A. Schmid, "Persistent currents in mesoscopic rings by suppression of charge fluctuations," Phys. Rev. Lett. 66, 80-83 (1991).

${ }^{202}$ H. Bouchiat, "New clues in the mystery of persistent currents," Physics $\mathbf{1}$, 7 (2008).

${ }^{203}$ L. P. Lévy, G. Dolan, J. Dunsmuir, and H. Bouchiat, "Magnetization of mesoscopic copper rings: Evidence for persistent currents," Phys. Rev. Lett. 64, 2074-2077 (1990).

${ }^{204}$ V. Chandrasekhar et al., "Magnetic response of a single, isolated gold loop," Phys. Rev. Lett. 67, 3578-3581 (1991).

${ }^{205}$ D. Mailly, C. Chapelier, and A. Benoit, "Experimental observation of persistent currents in GaAs-AlGaAs single loop," Phys. Rev. Lett. 70, 2020-2023 (1993).

${ }^{206} \mathrm{~W}$. Rabaud et al., "Persistent currents in mesoscopic connected rings," Phys. Rev. Lett. 86, 3124-3127 (2001).

${ }^{207}$ N. A. J. M. Kleemans et al., "Oscillatory persistent currents in selfassembled quantum rings," Phys. Rev. Lett. 99, 146808 (2007).

${ }^{208}$ A. C. Bleszynski-Jayich et al., "Persistent currents in normal metal rings," Science 326, 272-275 (2009).

${ }^{209}$ H. Bluhm, N. C. Koshnick, J. A. Bert, M. E. Huber, and K. A. Moler, "Persistent currents in normal metal rings," Phys. Rev. Lett. 102, 136802 (2009).

${ }^{210}$ M. A. Castellanos-Beltran, D. Q. Ngo, W. E. Shanks, A. B. Jayich, and J. G. E. Harris, "Measurement of the full distribution of persistent current in normal-metal rings," Phys. Rev. Lett. 110, 156801 (2013).

${ }^{211}$ H.-F. Cheung, Y. Gefen, E. K. Riedel, and W.-H. Shih, "Persistent currents in small one-dimensional metal rings," Phys. Rev. B 37, 6050-6062 (1988).

${ }^{212} \mathrm{~F}$. von Oppen and E. K. Riedel, "Average persistent current in a mesoscopic ring,” Phys. Rev. Lett. 66, 84-87 (1991).
${ }^{213}$ J. F. Weisz, R. Kishore, and F. V. Kusmartsev, "Persistent current in isolated mesoscopic rings,” Phys. Rev. B 49, 8126-8131 (1994).

${ }^{214}$ T. Chakraborty and P. Pietiläinen, "Persistent currents in a quantum ring: Effects of impurities and interactions," Phys. Rev. B 52, 1932-1935 (1995).

${ }^{215}$ Y. V. Pershin and C. Piermarocchi, "Persistent and radiation-induced currents in distorted quantum rings," Phys. Rev. B 72, 125348 (2005).

${ }^{216} \mathrm{~A}$. Bruno-Alfonso and A. Latgé, "Quantum rings of arbitrary shape and non-uniform width in a threading magnetic field," Phys. Rev. B 77, 205303 (2008).

${ }^{217} \mathrm{H}$. Bary-Soroker, O. Entin-Wohlman, and Y. Imry, "Persistent currents of noninteracting electrons in one-, two-, and three-dimensional thin rings," Phys. Rev. B 82, 144202 (2010).

${ }^{218}$ A. H. C. Neto, F. Guinea, and N. M. R. Peres, "Edge and surface states in the quantum Hall effect in graphene," Phys. Rev. B 73, 205408 (2006).

${ }^{219}$ D. S. L. Abergel, V. M. Apalkov, and T. Chakraborty, "Interplay between valley polarization and electron-electron interaction in a graphene ring," Phys. Rev. B 78, 193405 (2008).

${ }^{220}$ C. W. J. Beenakker, A. R. Akhmerov, P. Recher, and J. Tworzydło, "Correspondence between Andreev reflection and Klein tunneling in bipolar graphene," Phys. Rev. B 77, 075409 (2008).

${ }^{221}$ M. Zarenia, J. M. Pereira, F. M. Peeters, and G. A. Farias, "Electrostatically confined quantum rings in bilayer graphene," Nano Lett. 9, 4088-4092 (2009).

${ }^{222}$ R. Jackiw, A. I. Milstein, S.-Y. Pi, and I. S. Terekhov, "Induced current and Aharonov-Bohm effect in graphene," Phys. Rev. B 80, 033413 (2009).

${ }^{223}$ M. M. Ma, J. W. Ding, and N. Xu, "Odd-even width effect on persistent current in zigzag hexagonal graphene rings," Nanoscale 1, 387-390 (2009).

${ }^{224}$ M. Zarenia, J. M. Pereira, A. Chaves, F. M. Peeters, and G. A. Farias, "Simplified model for the energy levels of quantum rings in single layer and bilayer graphene," Phys. Rev. B 81, 045431 (2010).

${ }^{225}$ D. Soriano and J. Fernández-Rossier, "Spontaneous persistent currents in a quantum spin Hall insulator," Phys. Rev. B 82, 161302 (2010).

${ }^{226}$ B.-L. Huang, M.-C. Chang, and C.-Y. Mou, "Persistent currents in a graphene ring with armchair edges," J. Phys.: Condens. Matter 24, 245304 (2012).

${ }^{227}$ D. Faria, A. Latgé, S. E. Ulloa, and N. Sandler, "Currents and pseudomagnetic fields in strained graphene rings," Phys. Rev. B 87, 241403 (2013).

${ }^{228}$ N. Bolívar, E. Medina, and B. Berche, "Persistent charge and spin currents in the long-wavelength regime for graphene rings," Phys. Rev. B 89, 125413 (2014).

${ }^{229} \mathrm{P}$. Michetti and P. Recher, "Bound states and persistent currents in topological insulator rings,” Phys. Rev. B 83, 125420 (2011).

${ }^{230}$ D. Sticlet, B. Dóra, and J. Cayssol, "Persistent currents in Dirac fermion rings," Phys. Rev. B 88, 205401 (2013).

${ }^{231}$ J. U. Nöckel, A. D. Stone, G. Chen, H. L. Grossman, and R. K. Chang, "Directional emission from asymmetric resonant cavities," Opt. Lett. 21, 1609-1611 (1996).

${ }^{232}$ C. Gmachl et al., "High-power directional emission from microlasers with chaotic resonators," Science 280, 1556-1564 (1998).

${ }^{233}$ S. M. Spillane, T. J. Kippenberg, and K. J. Vahala, "Ultralow-threshold raman laser using a spherical dielectric microcavity," Nature 415, 621-623 (2002).

${ }^{234}$ K. J. Vahala, “Optical microcavities," Nature 424, 839-846 (2003).

${ }^{235} \mathrm{G}$. Reecht et al., "Oligothiophene nanorings as electron resonators for whispering gallery modes," Phys. Rev. Lett. 110, 056802 (2013).

${ }^{236}$ L. Ying and Y.-C. Lai, "Robustness of persistent currents in twodimensional dirac systems with disorder," Phys. Rev. B 96, 165407 (2017).

${ }^{237}$ L. Huang, H.-Y. Xu, Y.-C. Lai, and C. Grebogi, "Level spacing statistics for two-dimensional massless Dirac billiards," Chin. Phys. B 23, 070507 (2014).

${ }^{238} \mathrm{P}$. Yu et al., "Gaussian orthogonal ensemble statistics in graphene billiards with the shape of classically integrable billiards," Phys. Rev. E 94, 062214 (2016).

${ }^{239}$ L. Brey and H. A. Fertig, "Electronic states of graphene nanoribbons studied with the Dirac equation," Phys. Rev. B 73, 235411 (2006).

${ }^{240} \mathrm{~L}$. A. Ponomarenko et al., "Chaotic Dirac billiard in graphene quantum dots," Science 320, 356-358 (2008). 
${ }^{241}$ C. Stampfer et al., "Tunable graphene single electron transistor," Nano Lett. 8, 2378-2383 (2008).

${ }^{242} \mathrm{~J}$. Wurm et al., "Symmetry classes in graphene quantum dots: Universal spectral statistics, weak localization, and conductance fluctuations," Phys. Rev. Lett. 102, 056806 (2009).

${ }^{243}$ C. W. J. Beenakker, "Colloquium: Andreev reflection and Klein tunneling in graphene,” Rev. Mod. Phys. 80, 1337-1354 (2008).

${ }^{244}$ H. Suzuura and T. Ando, "Crossover from symplectic to orthogonal class in a two-dimensional honeycomb lattice," Phys. Rev. Lett. 89, 266603 (2002).

${ }^{245} \mathrm{~S}$. Bittner et al., "Observation of a Dirac point in microwave experiments with a photonic crystal modeling graphene," Phys. Rev. B 82, 014301 (2010).

${ }^{246}$ S. Bittner, B. Dietz, M. Miski-Oglu, and A. Richter, "Extremal transmission through a microwave photonic crystal and the observation of edge states in a rectangular Dirac billiard,” Phys. Rev. B 85, 064301 (2012).
${ }^{247}$ B. Dietz, T. Klaus, M. Miski-Oglu, and A. Richter, "Spectral properties of superconducting microwave photonic crystals modeling Dirac billiards," Phys. Rev. B 91, 035411 (2015).

${ }^{248} \mathrm{~B}$. Dietz and A. Richter, "Quantum and wave dynamical chaos in superconducting microwave billiards," Chaos 25, 097601-097601 (2015).

${ }^{249}$ B. Dietz et al., "Spectral properties of Dirac billiards at the van Hove singularities," Phys. Rev. Lett. 116, 023901 (2016).

${ }^{250}$ L. Huang, R. Yang, and Y.-C. Lai, "Geometry-dependent conductance oscillations in graphene quantum dots," Europhys. Lett. 94, 58003 (2011).

${ }^{251}$ G. W. Semenoff, "Condensed-matter simulation of a three-dimensional anomaly," Phys. Rev. Lett. 53, 2449-2452 (1984).

${ }^{252}$ F. D. M. Haldane, "Model for a quantum Hall effect without Landau levels: Condensed-matter realization of the parity anomaly," Phys. Rev. Lett. 61, 2015-2018 (1988). 